University of Nebraska - Lincoln

DigitalCommons@University of Nebraska - Lincoln

U.S. Air Force Research

U.S. Department of Defense

2014

\title{
Unsteady aerodynamics modeling for aircraft maneuvers: A new approach using time-dependent surrogate modeling
}

Mehdi Ghoreyshi

U.S. Air Force Academy, Mehdi.Ghoreyshi@usafa.edu

Russell M. Cummings

USAF Academy, russ.cummings@usafa.edu

Follow this and additional works at: http:// digitalcommons.unl.edu/usafresearch

Ghoreyshi, Mehdi and Cummings, Russell M., "Unsteady aerodynamics modeling for aircraft maneuvers: A new approach using timedependent surrogate modeling" (2014). U.S. Air Force Research. 56.

http://digitalcommons.unl.edu/usafresearch/56

This Article is brought to you for free and open access by the U.S. Department of Defense at DigitalCommons@University of Nebraska - Lincoln. It has been accepted for inclusion in U.S. Air Force Research by an authorized administrator of DigitalCommons@University of Nebraska - Lincoln. 


\title{
Unsteady aerodynamics modeling for aircraft maneuvers: A new approach using time-dependent surrogate modeling
}

\author{
Mehdi Ghoreyshi*, Russell M. Cummings \\ High Performance Computing Research Center, U.S. Air Force Academy, USAF Academy, CO 80840-6400, USA
}

\section{A R T I C L E I N F O}

\section{Article history:}

Received 10 September 2013

Received in revised form 13 May 2014

Accepted 28 September 2014

Available online 6 October 2014

\section{Keywords:}

Indicial functions

Kriging

Unsteady aerodynamics

Optimal control

Dynamic derivatives

\begin{abstract}
A B S T R A C T
A new approach for computing the unsteady and nonlinear aerodynamic loads acting on a maneuvering aircraft is presented based on linear and nonlinear indicial response methods. The novelty of this approach relies on the use of a grid motion technique for CFD calculation of response functions and the development of a time-dependent surrogate model that fits the relationship between flight conditions (Mach number and angle of attack) and responses calculated from a limited number of simulations (samples). The reduced-order model, along with the surrogate model, provides a means for rapid calculation of response functions and predicting aerodynamic forces and moments during maneuvering flight. The maneuvers are generated using a time-optimal prediction code, each covering a different range of angle of attack and motion rates. The side-slip angle ranges from $-5^{\circ}$ to $5^{\circ}$ for all maneuvers, and the model assumes that the lateral aerodynamics is linear with side-slip angle over this range. Results presented show that the aircraft studied in the current paper exhibits highly nonlinear roll moments even at low angles of attack which the linear model fails to predict. The results of the new model provide some evidence that, for a certain range of input parameters, in certain maneuvers considered, the predictions match quite well with URANS CFD predictions. The models were at least better than traditional quasisteady predictions. However, for aircraft maneuvering at high angles of attacks, discrepancies are found in lateral coefficients between the model and CFD. At these conditions, the lateral airloads become highly nonlinear with side-slip angle and the model fails to predict these effects. Also, the results show that the CFD calculation of response functions in the high angle of attack flight regime remains a challenging task.
\end{abstract}

Published by Elsevier Masson SAS.

\section{Introduction}

Computational Fluid Dynamics (CFD) tools have become credible for the computation of aerodynamics experienced by a maneuvering fighter with time history effects. This allows for CFD to reduce the amount of wind tunnel and flight testing time required for aircraft development. At the highest practical level, a full-order aerodynamic model can be developed based on the direct solution of the discretized Reynolds-Averaged Navier-Stokes (RANS) equations coupled with the dynamic equations governing aircraft motion [27]. First attempts at this approach were limited to twodimensional test cases, while with recent advances in computing techniques and the capabilities provided by high performance computing resources, the coupled CFD-flight dynamics of a full aircraft has been studied $[39,14]$. However, full-order modeling is an

\footnotetext{
* Corresponding author.

E-mail addresses: Mehdi.Ghoreyshi@usafa.edu (M. Ghoreyshi), Russ.Cummings@usafa.edu (R.M. Cummings).
}

infinite-dimensional problem because the solution at each time depends on all of the states at times prior to the current state and the flow equations describe the motion of the fluid at infinitely many points [27,47]. Also, an aerodynamic model for stability and control requires a large number of coupled computations for different values of motion frequency and amplitude which makes full-order simulation a very expensive approach.

To make timely progress in the use of CFD for aircraft design, efforts over the last few years have been spent mainly on the development of a Reduced Order Model (ROM) using CFD from an appropriate training maneuver(s) and an accurate System IDentification (SID) approach [28,30,5]. The objective of the ROMs is to develop a model that significantly reduces the CFD simulation time required to create a full aerodynamics database, making it possible to accurately model aircraft static and dynamic characteristics (within the range of data used for model generation) from a number of time-accurate CFD simulations. These models need an initial or upfront cost to estimate, or identify, the unknown parameters. Once the model has been created, however, the aerodynamics 


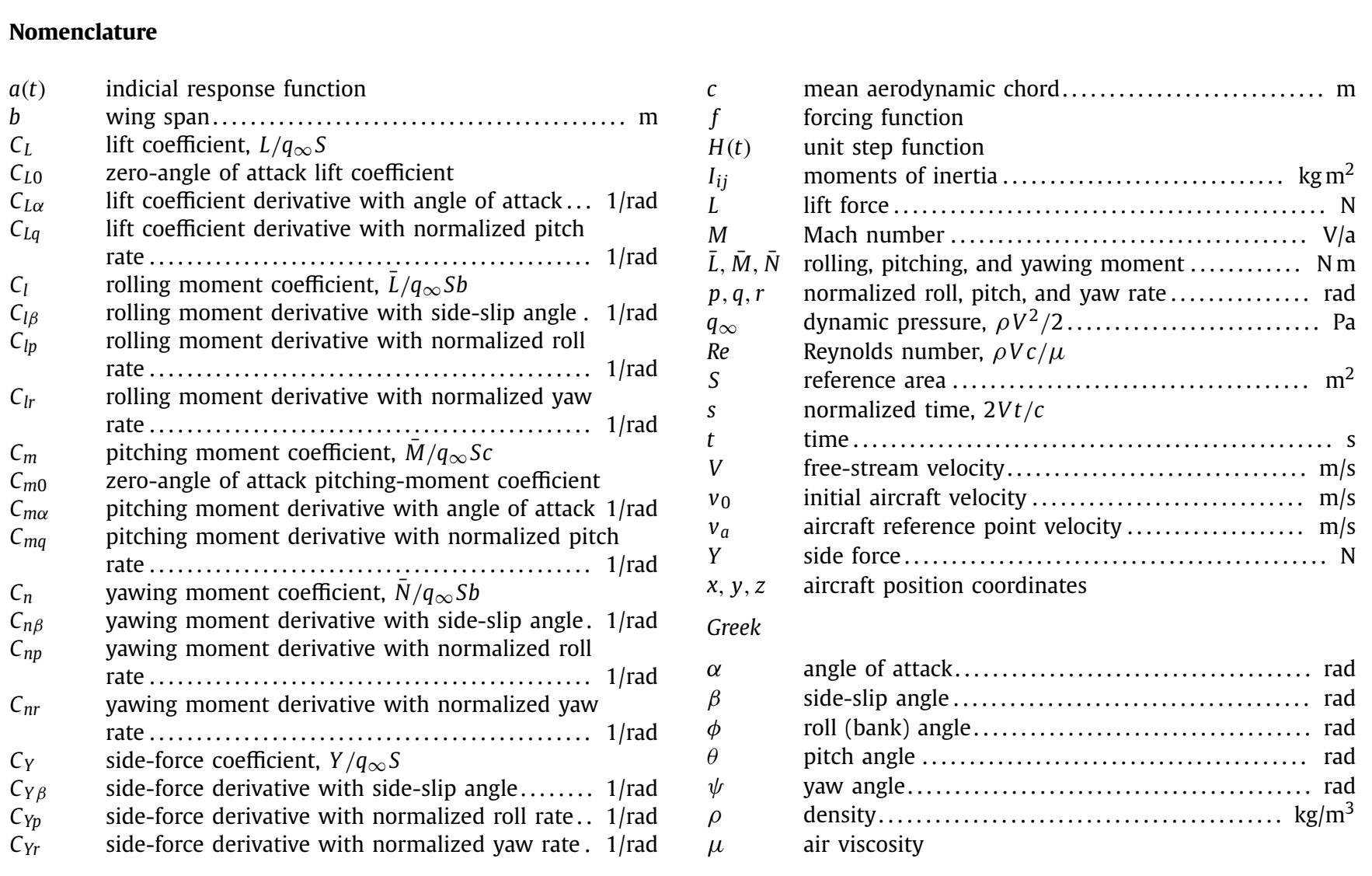

prediction of a wide range of maneuvers can be determined in order of a few seconds.

ROMs can be grouped into two different categories of parametric and nonparametric depending on the system identification method used. The parametric types provide a structure for representing aerodynamic forces and moments in the aircraft equations of motion. On the other hand, nonparametric models are concerned with the measured input/output behavior of the aircraft dynamics. The current paper aims to assess the accuracy of predictions of a parametric reduced order model based on indicial response method of Tobak [43].

The transient aerodynamic response due to a unit step change in a forcing parameter, such as angle of attack or pitch rate is a so-called "indicial function". A distinction should be made between indicial and response functions; a response corresponds to the response of a system to a general input, but an indicial response is the specific system response due to a unit step change in the input (such as angle of attack). Assuming that the indicial functions are known, the linear aerodynamic forces and moments induced in any maneuver can be estimated using the well-known Duhamel's superposition integral [26]. Note that aerodynamic predictions by using Duhamel's integral are only valid for linear regimes of flow. To overcome this problem, Tobak [43,47] formulated a nonlinear indicial response model for predicting aerodynamic responses to an arbitrary angle of attack variation. These models have then been used as a fundamental approach to represent the unsteady aerodynamic loads, in particular for two-dimensional airfoils. There have been only limited reports of using these models for aerodynamics modeling of three-dimensional configurations due to limitations of the identification methods of response functions for aircraft configurations. Among these works are the well-known studies of Klein and Norderer [22,23] who applied the indicial response method to an aircraft small-amplitude motion around a trim point. Klein and Murphy [21] and Pamadi et al. [31] later extended this model for aerodynamic modeling of the F-16XL aircraft at high angles of attack. They approximated aircraft responses (including indicial responses) by exponential functions and then identified the unknowns using wind tunnel and flight test data. However, an exponential function is not valid to represent the initial behavior of response functions. Also, wind tunnel and flight test data are expensive and typically only available late in the aircraft design cycle.

Recently, CFD solutions for the indicial response of airfoils and wings have been reported (see for example, Singh and Baeder [40] and Raveh [32]). Also, Ghoreyshi et al. [15] described an approach based on a grid motion technique for CFD-type calculation of linear and nonlinear response functions with respect to angle of attack and pitch rate. Ghoreyshi and Cummings [12] later used this approach to generate indicial functions due to longitudinal and lateral forcing parameters of a generic unmanned combat air vehicle (UCAV) and used these functions for predicting the unsteady aerodynamic responses to aircraft six degrees of freedom maneuvers. They showed that while unsteady lift, side-force and pitch moment (all estimated from indicial response methods) match quite well with full-order simulations in the linear regime, the roll and yaw moments (again estimated from indicial response methods) do not match even at low angles of attack. For the vehicle studied, the roll and yaw moment variation with the angle of attack and Mach number is highly nonlinear [6]. The objective of this paper is to develop a framework for approximating time-dependent response (including indicial) functions in the input design space (angle of attack/Mach). This framework allows rapid calculation of response functions and predicting aerodynamic forces and moments during maneuvering flight.

Having a ROM to predict the aerodynamic responses to any arbitrary motion over a wide flight regime could become a very expensive approach because a large number of response func- 
tions need to be computed for each combination of angle of attack and free-stream Mach number. Typically, the CFD simulation of response functions start from a steady state solution and are marched (iterated) in pseudo time within each physical time step using a dual-time stepping scheme. The generation of all response functions in the angle of attack/Mach number space using CFD is therefore expensive and makes the creation of a ROM very time consuming. However, these models are still cheaper than full-order simulations because the ROMs based on response functions eliminate the need to repeat calculations for each frequency. In this paper, a surrogate model is proposed based on the Kriging technique [11] to model response functions for new flight conditions from available (observed) responses. These observed responses are viewed as a set of time-correlated spatial processes where the output is considered a time-dependent function.

The present study develops a reduced order model based on the linear and nonlinear indicial response methods for computing the aerodynamic loads acting on a maneuvering aircraft. The response functions include longitudinal and lateral forces and moments and are directly calculated using unsteady RANS simulations with a grid motion technique. An important feature of this technique is uncoupling the effects of angle of attack/side-slip and angular rates from pitch-yaw-roll motions. A method to efficiently reduce the number of response calculations is proposed. This method uses a time-dependent surrogate model to fit the relationship between flight conditions and response functions from a limited number of response simulations (samples). The six-degreeof-freedom (6-DOF) aerodynamics model is then created with predicted response functions at each time instant using the developed surrogate model. The model will then be evaluated for several maneuvers, which were replayed directly through an unsteady CFD simulation.

Note that only one CFD code and only one turbulence model has been used throughout the study based on our experience with these tools in predicting unsteady aerodynamics. Since the primary result of the work is to validate the modeling approaches, only comparisons will be made between the model results and the original CFD simulations. The following assumptions were made in this study. The unsteady lift and pitch moment were predicted from response functions with respect to angle of attack and pitch rate only; the effects of rate of change of velocity, i.e. $\dot{V}$ were not considered. Also, the unsteady effects in drag force were assumed to be small and therefore were not discussed. The angle of attack response functions were assumed to change with angle of attack and Mach number but not with side-slip angle. Also, it was assumed that pitch rate response functions change only with changes in free-stream Mach number but do not vary with the changes in angle of attack for the maneuvers studied. The lateral airloads were assumed only depend on side-slip angle and angular rates of roll and yaw. Again the angular rate indicial functions change only with Mach number. The side-slip angle range was from $-5^{\circ}$ to $5^{\circ}$ for all maneuvers, and it was assumed that lateral aerodynamics is linear with side-slip angle over this range. Maneuvers were generated from a time-optimal prediction code using a derivativebased aerodynamic model. In these maneuvers, the angular rates at the initial time were assumed to be zero. Static and dynamic derivatives were estimated from response function values at the final time, where the response function reaches a steady-state solution. The control derivatives (estimated from previous studies) were used in the generation of maneuvers but the replay of maneuvers in CFD did not move control surfaces. Static tables were also generated from steady-state CFD solutions at different flight conditions. Tables along with estimated dynamic derivatives provided quasi-steady aerodynamic predictions. Finally, the relationship between response functions and input parameters (angle of attack and Mach number) was approximated by a time-dependent surrogate model that assumes responses as a set of time-correlated spatial processes.

\section{Formulation}

\subsection{CFD solver}

The flow solver used for this study is the Cobalt code [42] that solves the unsteady, three-dimensional and compressible NavierStokes equations in an inertial reference frame. It is important to consider that this frame is different from an inertial frame usually attached to the Earth's center and body-fixed frame used in flight dynamics analysis. In these inertial grid coordinates, the $x$ and $z$ axes have opposite directions to those in the body-axes system. Therefore, careful consideration will need to be given to apply the aircraft rotations and translations into grid motions. In Cobalt, the Navier-Stokes equations are discretized on arbitrary grid topologies using a cell-centered finite volume method. Second-order accuracy in space is achieved using the exact Riemann solver of Gottlieb and Groth [17], and least squares gradient calculations using QR factorization. To accelerate the solution of discretized system, a point-implicit method using analytic first-order inviscid and viscous Jacobians. A Newtonian sub-iteration method is used to improve time accuracy of the point-implicit method. Tomaro et al. [48] converted the code from explicit to implicit, enabling Courant-Friedrichs-Lewy numbers as high as $10^{6}$.

\subsection{Indicial response theory}

Linear and nonlinear indicial response methods are detailed for aerodynamic loads prediction of a maneuvering aircraft. The indicial response of a linear system, denoted by $a(t)$, is the system response to a unit step change in the forcing parameter. The unit step function is denoted by $H(t)$ and is defined as:

$H(t)= \begin{cases}1 & \text { for } t \geq 0 \\ 0 & \text { for } t<0\end{cases}$

The general response of the linear system is related to the forcing parameter of $f$ using Duhamel's superposition integral (or convolution integral) as detailed by Duffy [7]:

$y(t)=f(0) a(t)+\int_{0}^{t} \frac{d f(\tau)}{d \tau} a(t-\tau) d \tau$

where $f(0)$ shows the forcing parameter value at time zero. Findeisen [9] used "differential theorem of the convolution integral" and showed that:

$$
\begin{aligned}
y(t) & =f(0) a(t)+\int_{0}^{t} \frac{d f(\tau)}{d \tau} a(t-\tau) d \tau \\
& =\frac{d}{d t}\left[\int_{0}^{t} f(\tau) a(t-\tau) d \tau\right]
\end{aligned}
$$

In the above approach $y=0$ for $f(t)=0$. For a non-excited system that has a non-zero initial value, the equation changes to:

$$
\begin{aligned}
y(t) & =y_{0}+f(0) a(t)+\int_{0}^{t} \frac{d f(\tau)}{d \tau} a(t-\tau) d \tau \\
& =y_{0}+\frac{d}{d t}\left[\int_{0}^{t} f(\tau) a(t-\tau) d \tau\right]
\end{aligned}
$$


where $y_{0}$ shows system value by setting $f(t)=0$. These equations have been used to predict unsteady aerodynamic forces and moments as well [46,45]. To represent the unsteady lift and pitch moment using indicial method, it is assumed that they depend on angle of attack and pitch rate. Indicial responses due to a unit step change in angle of attack, $\alpha$, and normalized pitch rate, $q$, are denoted by $C_{j_{\alpha}}$ and $C_{j_{q}}$, respectively; where $C_{j}=\left[C_{L}, C_{m}\right]$ represent lift and pitch moment coefficients. The unsteady lift and pitch moment are then calculated by adding Duhamel's integrals with respect to $\alpha$ and $q$, i.e.

$$
\begin{aligned}
C_{j}(t)= & C_{j_{0}}+\alpha(0) C_{j \alpha}(t)+\int_{0}^{t} C_{j \alpha}(t-\tau) \frac{d \alpha(\tau)}{d \tau} d \tau \\
& +\int_{0}^{t} C_{j q}(t-\tau) \frac{d q(\tau)}{d \tau} d \tau
\end{aligned}
$$

where $C_{j_{0}}$ denote the zero-angle of attack lift and pitch moment coefficients and are found from static calculations; $\alpha(0)$ is the angle of attack at time zero or the initial time of maneuver. Note that $\alpha(0) C_{j \alpha}(t)$ is different from $C_{j_{0}}$. The maneuvers in this study start from a steady-state solution with $q(0)=0$ and therefore the term of $q(0) C_{j q}(t)$ was not added to the equation. Using "differential theorem of the convolution integral", Eq. (5) changes to:

$$
\begin{aligned}
C_{j}(t)= & C_{j_{0}}+\frac{d}{d t}\left[\int_{0}^{t} C_{j \alpha}(t-\tau) \alpha(\tau) d \tau\right] \\
& +\frac{d}{d t}\left[\int_{0}^{t} C_{j q}(t-\tau) q(\tau) d \tau\right]
\end{aligned}
$$

These equations predict lift and pitch moment responses in the linear regime of flow.

Tobak [44] extended this model to a nonlinear situation by calculating indicial functions at different angles of attack and then used the model in predicting aerodynamic responses to pitching motions. Ghoreyshi and Cummings [12] later extended this model to include lateral aerodynamic coefficients and generated the response functions at different angle of attack and free-stream Mach numbers. They showed that the roll and yaw moments (estimated from indicial response methods) do not match even at low angles of attack due to linear assumptions in the model. In this study, a nonlinear model is considered where the responses in the angle of attack and side-slip depend on both the angle of attack and Mach number. It is assumed that the response functions with respect to the angular rates change with changes in free-stream Mach number but do not vary with the changes in angle of attack for the maneuvers studied. This is a reasonable assumption for low to moderate angles of attack range based on aerodynamic prediction methods described in aircraft design textbooks [37]. The unsteady lift and pitch moment coefficients at time $t$ are obtained using nonlinear indicial response theory as:

$$
\begin{aligned}
C_{j}(t)= & C_{j_{0}}(M)+\alpha(0) C_{j \alpha}(t, \alpha, M) \\
& +\int_{0}^{t} C_{j \alpha}(t-\tau, \alpha, M) \frac{d \alpha(\tau)}{d \tau} d \tau \\
& +\int_{0}^{t} C_{j q}(t-\tau, M) \frac{d q(\tau)}{d \tau} d \tau
\end{aligned}
$$

where $M$ denotes the free-stream Mach number. The response functions due to pitch rate, i.e. $C_{j q}(M)$ for $j=L, m$ can be estimated by using a time-dependent interpolation scheme from the observed responses. This value is next used to estimate the second integral in Eq. (7), however, the estimation of nonlinear $C_{j \alpha}(t, \alpha, M)$ for $j=L, m$ needs more explanation. Assume a set of angle of attack samples of $\alpha=\left[\alpha_{1}, \alpha_{2}, \ldots, \alpha_{n}\right]$ at free-stream Mach numbers of $M=\left[M_{1}, M_{2}, \ldots, M_{m}\right]$, where the spacing can be uniform or non-uniform. The response functions at each angle of $\alpha_{i}, i=1,2, \ldots, n$ degrees and Mach number of $M_{k}, k=1,2, \ldots, m$ are calculated by holding the angle of attack fixed at $\alpha=\alpha_{i}$ degrees, and then performing a small step in the angle of attack to $\alpha=\alpha_{i}+\Delta \alpha$. The response functions are then computed by taking the differences between time-varying responses occurring after the step and the steady-state solution at $\alpha=\alpha_{i}$ degrees, and dividing them by the magnitude of the step [47].

Assuming that the lateral airloads only depend on side-slip angle $(\beta)$, normalized roll rate $(p)$, and normalized yaw rate $(r)$, the unsteady lateral forces and moments using indicial response method are written as:

$$
\begin{aligned}
C_{j}(t)= & \beta(0) C_{j \beta}(t, \alpha, M)+\int_{0}^{t} C_{j_{\beta}}(t-\tau, \alpha, M) \frac{d \beta(\tau)}{d \tau} d \tau \\
& +\int_{0}^{t} C_{j_{p}}(t-\tau, M) \frac{d p(\tau)}{d \tau} d \tau \\
& +\int_{0}^{t} C_{j_{r}}(t-\tau, M) \frac{d r(\tau)}{d \tau} d \tau
\end{aligned}
$$

where $j=Y, l, n$ denote the side-force, roll and yaw moments, respectively; $\beta(0)$ represent the side-slip angle at time zero (or initial time of maneuver). Again, it was assumed initialtime angular rates are zero i.e., $p(0)=r(0)=0$. The functions of $C_{L \alpha}(t, \alpha, M), C_{m_{\alpha}}(t, \alpha, M), C_{L q}(t, M), C_{m q}(t, M), C_{Y \beta}(t, \alpha, M)$, $C_{l \beta}(t, \alpha, M), \quad C_{n \beta}(t, \alpha, M), \quad C_{Y p}(t, M), \quad C_{l p}(t, M), \quad C_{n p}(t, M)$, $C_{Y r}(t, M), C_{l r}(t, M)$, and $C_{n r}(t, M)$ are unknown and should be determined.

The indicial functions can be estimated via analytical, experimental, or computational methods. Limited analytical expressions of indicial functions exist for two-dimensional airfoils [2]. However, these analytical expressions are not valid for aircraft configurations due to the three-dimensional tip vortices. Experimental tests are practically nonexistent for indicial function measurements due to wind tunnel constraints. The most common way of calculating the indicial functions from experimental data is by using harmonic (oscillatory) motions. For example, Reisenthel et al. [35] and Reisenthel and Bettencourt $[33,34]$ used this method to approximate the nonlinear indicial functions of a $65^{\circ}$ delta wing. However, the derived indicial functions using harmonic motions depend largely on the quality of motion, e.g. amplitude and frequency.

CFD offers a viable method to estimate 3D indicial functions but in the absence of credible wind tunnel test data, it is difficult to validate CFD predictions. Also, special considerations are required to simulate step responses in CFD. Singh and Baeder [40] used a surface transpiration approach to directly calculate the angle of attack indicial response using CFD. Ghoreyshi et al. [15] also described an approach based on a grid motion technique for CFD-type calculation of linear and nonlinear response functions. In this paper, the response functions due to longitudinal and lateral forcing parameters (angle of attack, side-slip angle, and angular rates) are calculated using CFD and the grid motion approach. 
Cobalt uses an arbitrary Lagrangian-Eulerian formulation and hence allows all translational and rotational degrees of freedom [15]. The code can simulate both free and specified six degree of freedom $(6 \mathrm{DoF})$ motions. The rigid motion is specified from a motion input file. For the rigid motion the location of a reference point on the aircraft is specified at each time step. In addition the rotation of the aircraft about this reference point is also defined using the rotation angles of yaw, pitch, and roll (bank). The aircraft reference point velocity, $v_{a}$, in an inertial frame is then calculated to achieve the required angles of attack and side-slip, and the forward speed. The velocity is then used to calculate the location. The initial aircraft velocity, $v_{0}$, is specified in terms of Mach number, angle of attack and side-slip angle in the main file. The instantaneous aircraft location for the motion file is then defined from the relative velocity vector, $v_{a}-v_{0}$.

\subsection{Surrogate-based modeling of indicial functions}

A multiple-state-variable model based on indicial functions requires a special time-dependent surrogate-based modeling approach that predicts indicial responses from available (observed) responses. In this paper, these observed responses are viewed as a set of time-correlated spatial processes where the output is considered a time-dependent function. Romero et al. [36] developed a framework for multi-stage Bayesian surrogate models for the design of time dependent systems and tested their model for free vibrations of a mass-spring-damper system assuming the input parameters of stiffness and damping factor at different initial conditions. This framework is examined for reduced order modeling of nonlinear and unsteady aerodynamic loads. Assume an input vector of $\mathbf{x}(t)=\left(x_{1}(t), x_{2}(t), \ldots, x_{n}(t)\right)$ where $n$ represents the dimensionality of the input vector. To construct a surrogate model for fitting the input-output relationship, the unsteady aerodynamic responses corresponding to a limited number of input parameters (training parameters or samples) need to be generated. Design of Experiment methods, for example, can be used to select $m$ samples from the input space. The input matrix $\mathbf{D}(m \times n)$ is then defined as:

$\mathbf{D}=\left[\begin{array}{cccc}x_{11} & x_{12} & \cdots & x_{1 n} \\ x_{21} & x_{22} & \cdots & x_{2 n} \\ \vdots & \vdots & \vdots & \vdots \\ x_{m 1} & x_{m 2} & \cdots & x_{m n}\end{array}\right]$

where rows correspond to different combinations of the design parameters. For each row in the input matrix, a time-dependent response was calculated at $p$ discrete values of time, and this information is summarized in the output matrix of $\mathbf{Z}(m \times p)$ as:

$\mathbf{Z}=\left[\begin{array}{cccc}y_{11} & y_{12} & \cdots & y_{1 p} \\ y_{21} & y_{22} & \cdots & y_{2 p} \\ \vdots & \vdots & \vdots & \vdots \\ y_{m 1} & y_{m 2} & \cdots & y_{m p}\end{array}\right]$

where for aerodynamic loads modeling, $p$ equals the number of iterations used in time-marching CFD calculations. The objective of surrogate modeling is to develop a model that allows predicting the aerodynamic response of $\mathbf{y}\left(\mathbf{x}_{\mathbf{0}}\right)=\left(y_{01}, y_{02}, \ldots, y_{0 p}\right)$ at a new combination of input parameter of $\mathbf{x}_{\mathbf{0}}$. To construct this surrogate model, the responses at each time step are assumed as a separate set, such that each column of the output matrix is a partial realization of the total response. In this sense, $p$ surrogate models are created; they are denoted as $\mathbf{Z}_{\mathbf{i}}(\mathbf{D})$ for $i=1,2, \ldots, p$. A universaltype Kriging function [11] is then used to approximate these models. For more details about creating Kriging models, the reader is
Table 1

Surrogate modeling parameters.

\begin{tabular}{ll}
\hline Parameter/vector of parameters & Value/content \\
\hline$n$ & 2 \\
$p$ & 1000 \\
$m$ & 69 \\
$\mathbf{x}$ & {$[\alpha, M]$} \\
$\mathbf{y}$ & {$\left[C_{L}, C_{m}, C_{Y}, C_{l}, C_{n}\right]$} \\
\hline
\end{tabular}

referred to Ghoreyshi et al. [16]. Having created Kriging models for each $\mathbf{Z}_{\mathbf{i}}(\mathbf{D})$ function, the total response at $\mathbf{x}_{\mathbf{0}}$ is then combination of predicted values of each model, i.e.

$\tilde{\mathbf{Z}}\left(\mathbf{x}_{\mathbf{0}}\right)=\left(\tilde{\mathbf{Z}}_{\mathbf{1}}\left(\mathbf{x}_{\mathbf{0}}\right), \tilde{\mathbf{Z}}_{\mathbf{2}}\left(\mathbf{x}_{\mathbf{0}}\right), \ldots, \tilde{\mathbf{Z}}_{\mathbf{p}}\left(\mathbf{x}_{\mathbf{0}}\right)\right)$

where, the tilde shows that Kriging model is an approximation of the actual function.

For the purpose of this study, the input matrix of $\mathbf{D}$ includes combinations of angle of attack and Mach number, i.e.

$\mathbf{D}=\left[\begin{array}{cc}\alpha_{11} & M_{12} \\ \alpha_{21} & M_{22} \\ \vdots & \vdots \\ \alpha_{m 1} & M_{m 2}\end{array}\right]$

this means $n=2$ and $\mathbf{x}_{0}=\left[\alpha_{0}, M_{0}\right]$. The output vector is also defined as $\mathbf{y}=\left[C_{L}, C_{m}, C_{Y}, C_{l}, C_{n}\right]$ and therefore output matrices of $\mathbf{Z}_{\mathbf{j}}$ are:

$\mathbf{Z}_{\mathbf{j}}=\left[\begin{array}{cccc}C_{j_{11}} & C_{j_{12}} & \cdots & C_{j_{1 p}} \\ C_{j_{21}} & C_{j_{22}} & \cdots & C_{j_{2 p}} \\ \vdots & \vdots & \vdots & \vdots \\ C_{j_{m 1}} & C_{j_{m 2}} & \cdots & C_{j_{m p}}\end{array}\right]$

where $j=[L, m, Y, l, n]$. Table 1 summarizes values of the parameters used.

\subsection{Time optimal maneuvers}

An optimal control approach $[1,18]$ is used to generate 6-DOF maneuvers for a generic UCAV. The feasible solutions are chosen based on the vehicle control and state constraints. This approach finds the optimal controls that transfer a system from the initial state to the final state while minimizing (or maximizing) a specified cost function [3]. In this paper, the initial and final states are fixed with trimmed flight conditions, but the rest of the maneuver is at out-of-trim conditions; the cost function is maneuver time.

The optimal control solver DIDO [38] is used here, which has been widely used and tested for aircraft optimal control problems. In DIDO, the total time history is divided into $N$ segments, spaced using a shifted Legendre-Gauss-Lobatto (LGL) rule $[4,20]$. The code uses Pseudo-Spectral (PS) methods for solving the optimal control problem. For an aircraft optimal time maneuver, the general 6-DOF aircraft equations of motion detailed in Etkin [8] serve as one of the constraints. The aircraft state vector consists of the position of the aircraft $(x, y, z)$, the standard Euler angles $(\phi, \theta, \psi)$, the velocity components in terms of Mach number and flow angles $(M, \alpha, \beta)$, and the body-axis components of the angular velocity vector $(p, q, r)$. (For additional details the reader is referred to the work of Ghoreyshi et al. [10].) Aircraft maneuvers are generated using a derivative-based aerodynamic model with estimated derivatives from static data and response function values at the final time (when the response reaches its steady-state solution). 


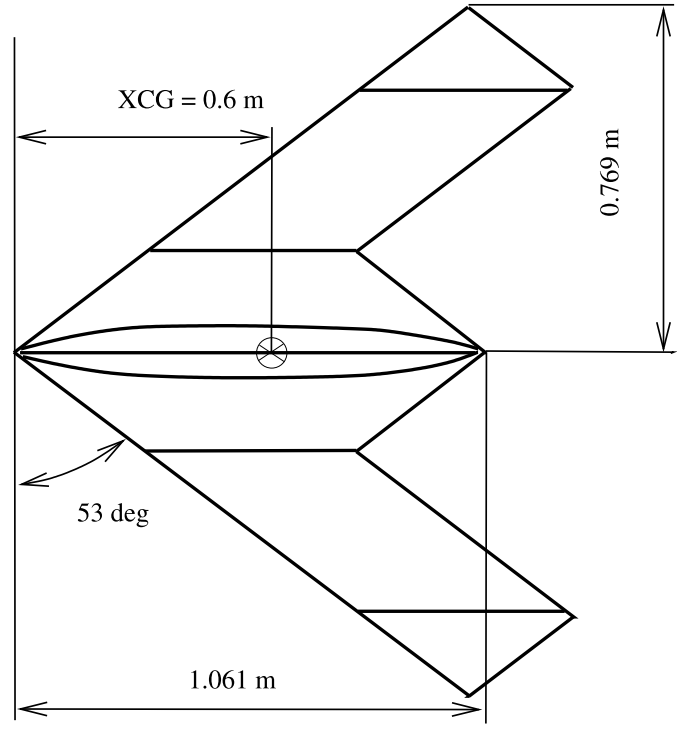

Fig. 1. UCAV SACCON geometry.

\section{Test case and validation}

A generic UCAV (Stability And Control CONfiguration, SACCON) shown in Fig. 1 is considered in this paper. The SACCON geometry and experimental data were available to the partners participating in NATO RTO Task Group AVT-161 (Assessment of Stability and Control prediction Methods for NATO Air and Sea Vehicles) [6]. The wind tunnel model was designed and manufactured at NASA Langley Research Center (LaRC). The model was designed to accommodate a belly sting mount for tests in the German-Dutch Low Speed Wing Tunnel (DNW-NWB) at DLR in Braunschweig and the $14^{\prime} \times 22^{\prime}$ low speed wind tunnel at NASA LaRC [29]. Two meshes are available: the first uses a belly mounted sting present in the experiments and the second has no sting. These grids are shown in Fig. 2 and contain around 9M points and 26M cells. The reader is referred to Ref. [15] for more details of geometry and meshes. CFD simulations were run on the Cray XE6 (open system) machine at the Engineering Research Development Center (ERDC) [the machine name is Chugach with $2.3 \mathrm{GHz}$ core speed and 11000 cores].

It should be noted, again, that this work does not investigate the grid resolution, numerical parameters, or turbulence modeling requirements for making accurate predictions. Therefore a fine grid has been generated and simulated using the Spalart-Allmaras (SA) [41] turbulence model based on our experience with SACCON CFD modeling. The coefficients of lift, drag, and pitch moment from Cobalt are compared with experiments in Fig. 2. These experiments were made at a Mach number of 0.1444 and Reynolds number of $1.61 \times 10^{6}$. Fig. 2 shows that the CFD predictions closely follow the trends of the experimental data up to moderate angles of attack. The offsets in the low angle of attack pitch moment in the model is likely due to the effects of the belly sting mounting present in the experiments [19]. Some of the SACCON aerodynamic features are shown in Fig. 3. Two vortices emanating from the wing tip and apex are present at $14^{\circ}$ angle of attack (Fig. 3(a)). These vortices lead to a negative pressure region on the upper wing surface and hence augment the lift force. As the angle of attack increases from $16^{\circ}$, the onset point of the outboard vortex starts to travel toward the wing apex due to increasing adverse pressure gradients. At $19.5^{\circ}$ angle of attack, the vortices are already interacting as shown in Fig. 3(b). Further increasing of the angle of attack causes the inboard vortex to start to breakdown (Fig. 3(c)). At higher angles of attack the tip vortex also breaks down. The interaction of the vortices produces a strong recirculation zone over the upper wing
(Fig. 3(d)) and results in wing stall and the aerodynamic center backward movement.

SACCON also has complicated aerodynamic characteristics at non-symmetric flow conditions. Some experimental aerodynamic behavior of the SACCON configuration in the lateral direction are shown in Fig. 4. The experimental results [6] show that side-force, yaw and roll moments are a nonlinear function of angle of attack, most significantly above angle of attack of $10^{\circ}$. Fig. 4 shows that the angle of attack-dependency can be seen in the roll moment even at low angles of attack. The lateral coefficients are nearly linear with side-slip angle for angles of attack below $15^{\circ}$, and become increasingly dependent on side-slip for angles of attack above $15^{\circ}$ as shown in Fig. 4.

For CFD simulations of a maneuvering SACCON, the mesh without the sting geometry was used. Also for the generation of SACCON maneuvers, the wind tunnel model was scaled up to fit the characteristics of a full size aircraft if this were to fly. Initially, estimations of the mass and moments of inertia were made, through work carried out in the NATO group, based on the Northrop YB-46 aircraft. Table 2 summarizes the SACCON flyable geometry parameters and mass and inertia.

\section{Results and discussion}

From the ROM equations given above, the unsteady aerodynamic responses to a wide range of aircraft maneuvers can be evaluated, although the model predictions are only valid within the range of input data used for the model generation. The model developed for this study could predict the variations in Mach number, angular rates, angle of attack, and side-slip angle during flight maneuvers. To check the accuracy of the new model, a linear ROM was created using indicial functions generated from a unit step change in input parameters. The look-up tables were generated from static CFD solutions in the input space. The dynamic derivatives were found from the solution of indicial functions with respect to $p, q, r$ at the final time of the response to step input, where the response reaches the steady-state solution. Tables with dynamic derivatives could predict nonlinear and quasi-steady aerodynamics, but not the unsteady effects. The table predictions were therefore used to highlight the unsteady effects in the solutions. Some of the prediction results are highlighted below.

\subsection{Calculation of indicial functions}

The input angle of attack for the SACCON maneuvers is in the range of $-5^{\circ}$ to $17^{\circ}$ with a Mach number range of 0.1 to 0.5 . The side-slip angle also ranges from $-5^{\circ}$ to $5^{\circ}$. The side-force, yaw and roll moments are nearly linear with side-slip for small values of side-slip and angles of attack below $15^{\circ}$ as shown in Fig. 4 . Also Fig. 5 shows the changes in experimental static lift and pitch moment with respect to the angle of attack in the presence of side-slip angles up to $5^{\circ}$. The Mach number and Reynolds number in these experiments are similar to those used in data shown in Fig. 2. These data show that lift is independent of side-slip angle for the angles of attack considered in this study. Fig. 5 also shows that pitch moment variations with side-slip angle are significant for angles of attack above $20^{\circ}$. Note that during high angles of attack maneuvers in this study, side slip angles values are smaller than side-slip values shown in the experimental data. Also a full factorial design was used in this study to select samples in the input space. The cost of a full-factorial design grows with the size of input space at a rate of $2^{n}$, where $n$ is the size of the input space. Therefore, the input space for the model creation only includes angle of attack and Mach number; side-slip is not included in the input space due to the high computational cost of modeling. 


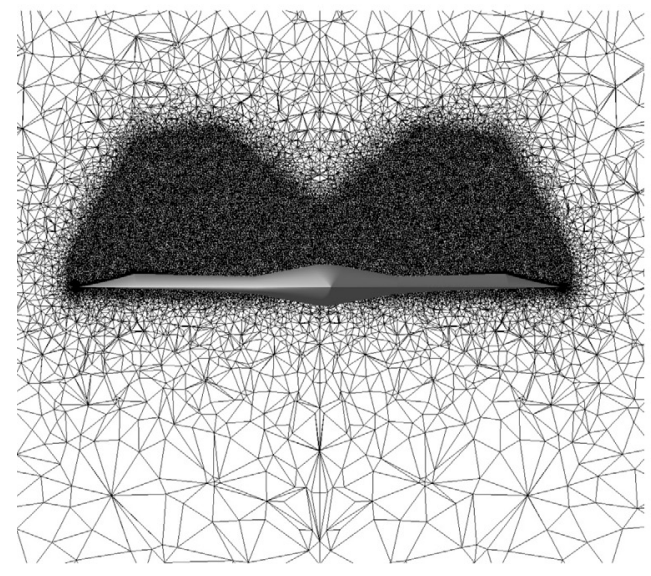

(a) grid without sting geometry.

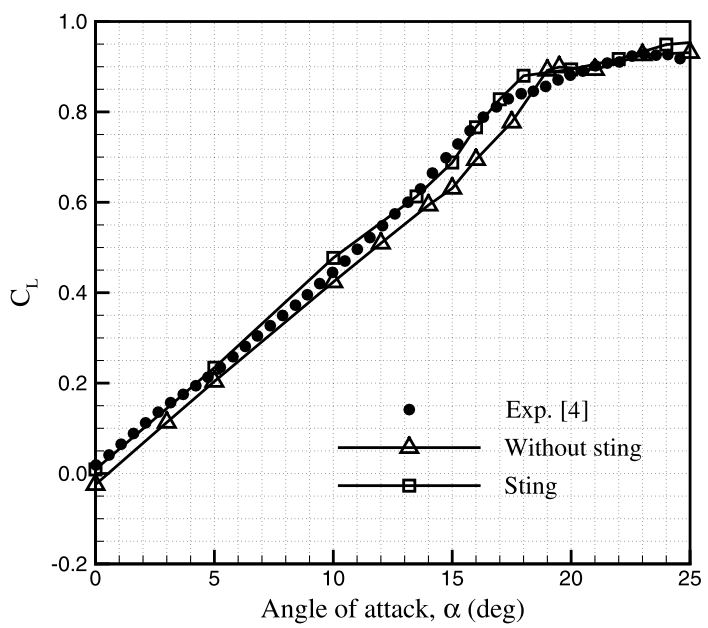

(c) Static lift coefficient

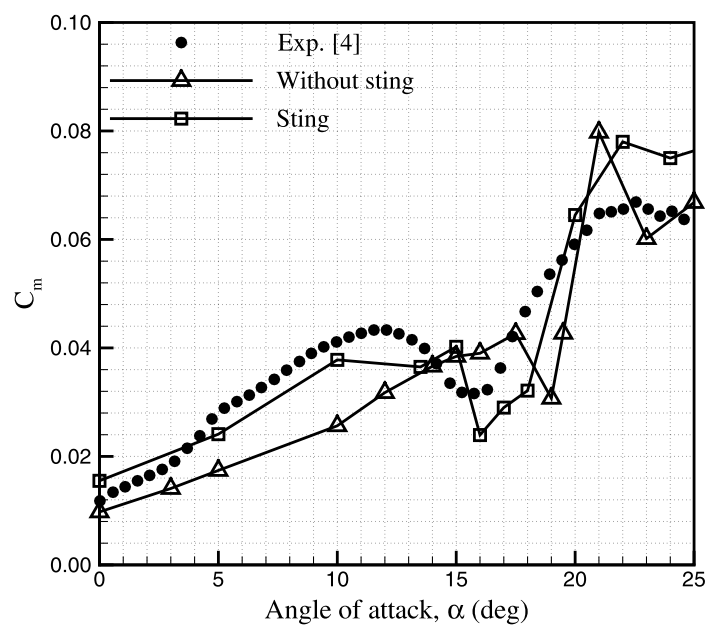

(e) Static pitch moment coefficient

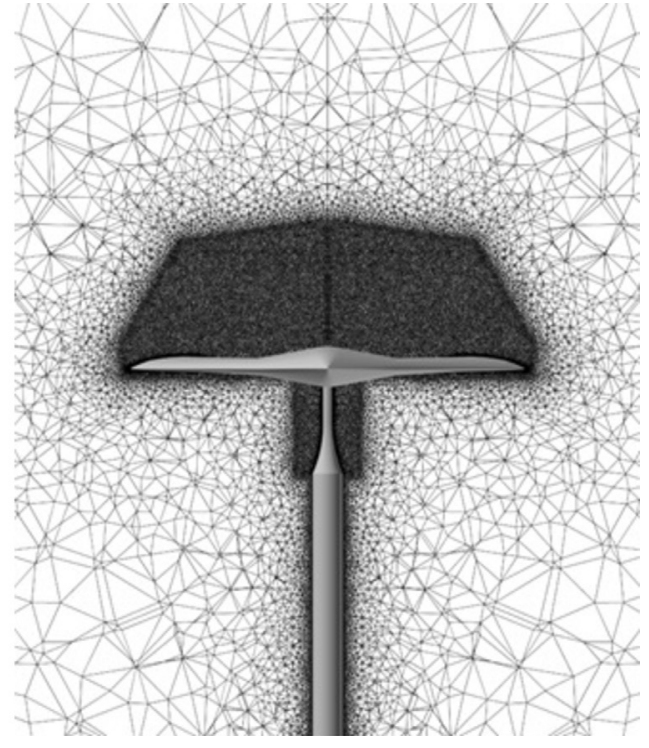

(b) grid with sting geometry

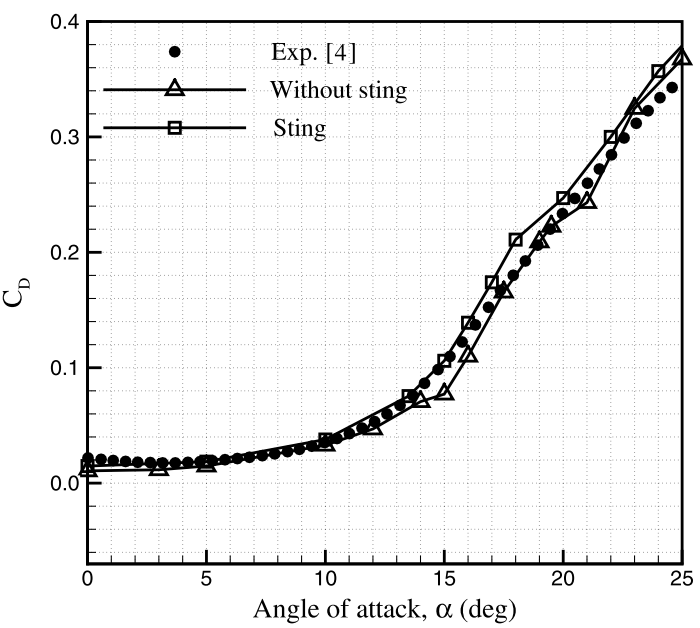

(d) Static drag coefficient

Fig. 2. The SACCON grids and static predictions at $M_{\infty}=0.144$ and $R e=1.61 \times 10^{6}$.

For surrogate modeling of response functions, a set of samples including 69 points is defined on the $\alpha$ and $M$ space using full factorial design. These points are uniformly spaced over $\alpha$ for Mach numbers of $0.1,0.3$, and 0.5 . In the present paper, the response functions are directly calculated from unsteady RANS simulations and using a grid motion tool. All computations started from a steady-state solution and then advanced in time using secondorder accuracy. The motion files were generated for step changes in 


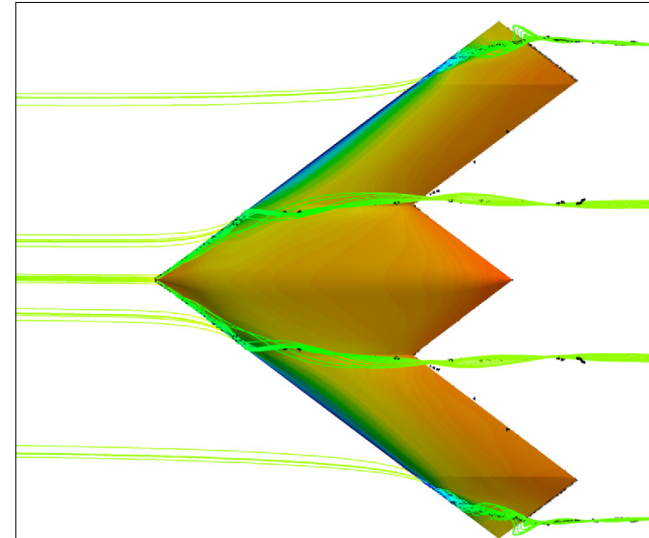

(a) $\alpha=14^{0}$

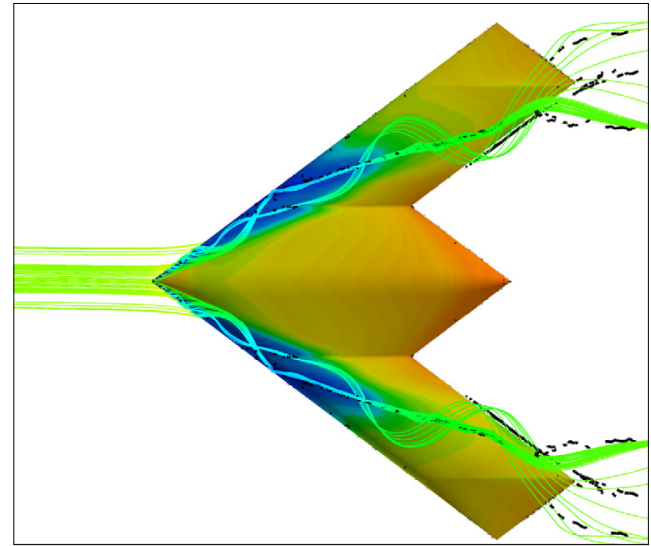

(c) $\alpha=20.5^{0}$

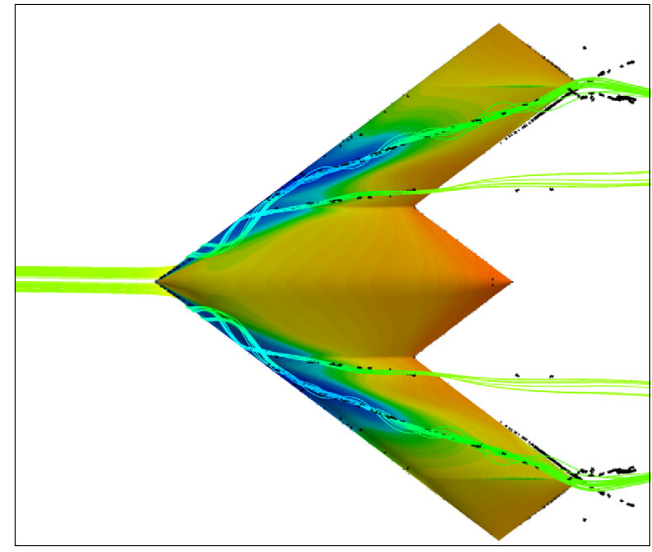

(b) $\alpha=19.5^{0}$

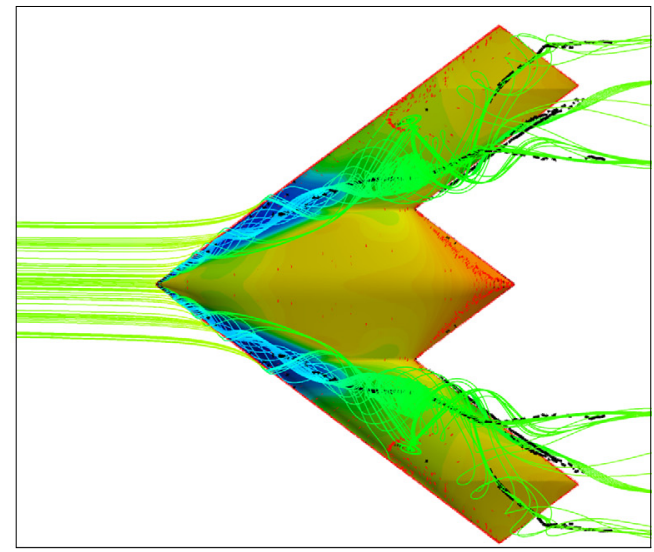

(d) $\alpha=23^{0}$

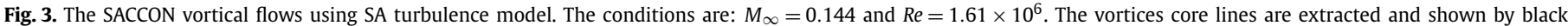

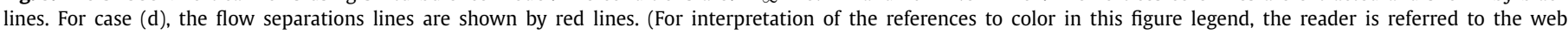
version of this article.)
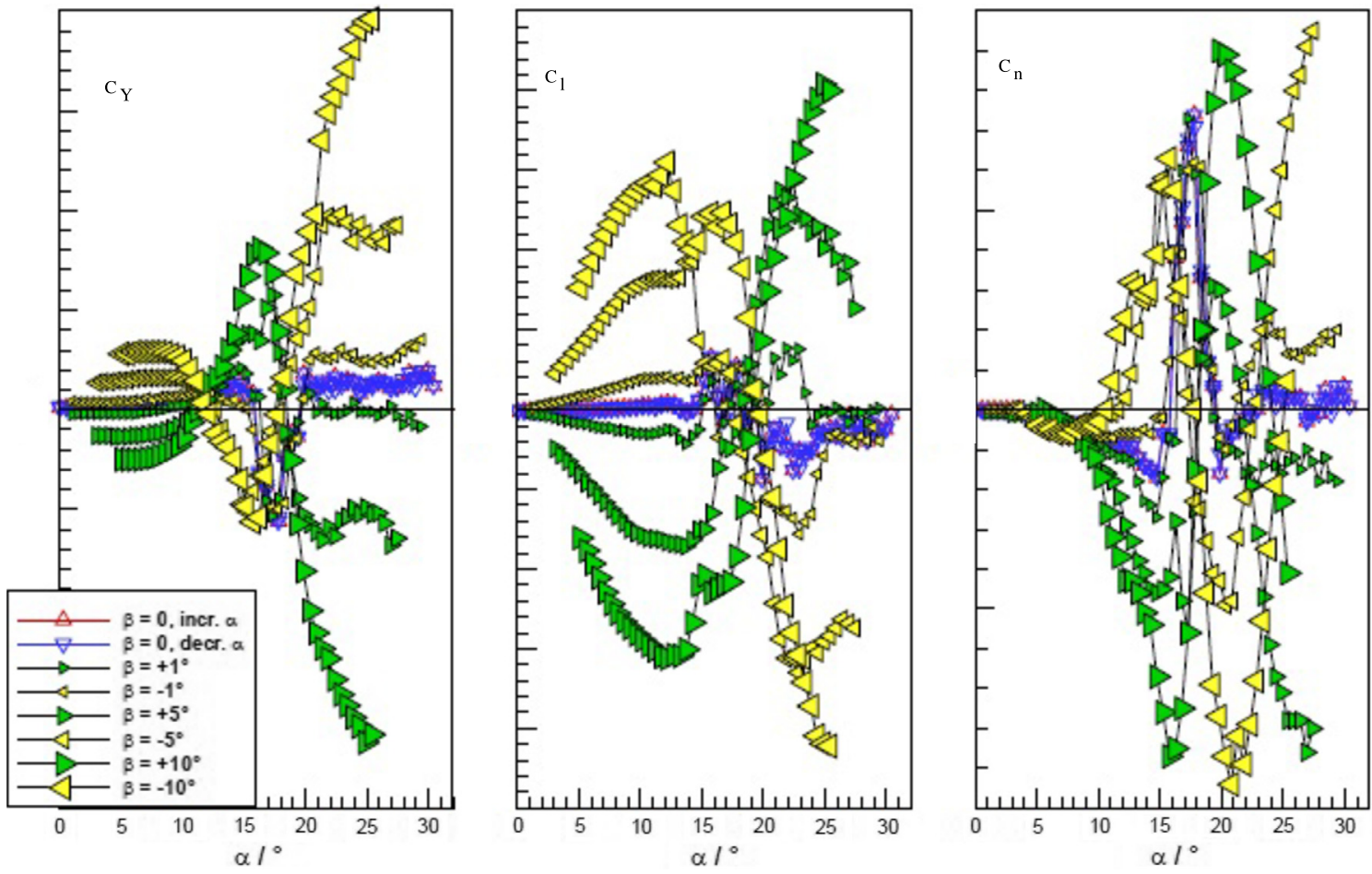

Fig. 4. SACCON side-force, roll-moment, yaw-moment versus angle of attack for different angles of side-slip. The figures were adapted from Ref. [6]. 
Table 2

Geometry parameters and mass/inertias of SACCON flyable model.

\begin{tabular}{lc}
\hline Mean aerodynamic chord, $\bar{c}(\mathrm{~m})$ & 5.011 \\
Wing area, $S\left(\mathrm{~m}^{2}\right)$ & 55.0 \\
Wing span, $b(\mathrm{~m})$ & 13.0 \\
$\mathrm{Ixx}\left(\mathrm{kg} \mathrm{m}^{2}\right)$ & 8014 \\
$\mathrm{Iyy}\left(\mathrm{kg} \mathrm{m}^{2}\right)$ & 6564 \\
$\mathrm{Izz}\left(\mathrm{kg} \mathrm{m}^{2}\right)$ & 8937 \\
Maximum take-off weight, MTWO $(\mathrm{kg})$ & 2000 \\
\hline
\end{tabular}

aircraft forcing parameters (angle of attack, side-slip angle, and angular rates). These files define the rotations and displacements at discrete time instants and Cobalt then interpolates motion data using cubic-splines and moves the grid for each computational time step. The grid undergoes only translation motion for $\alpha$ and $\beta$ responses, where the relative velocity between grid and flow at each instant defines the angle of attack and side-slip. For angular rate responses, the motions start from a steady-state solution with zero degrees angle of attack and side-slip angle. The grid then rotates and translates simultaneously. The rotation corresponds to a unit step change in the angular rate, while the translation motion is used in order to keep angles of attack and side-slip zero during rotations. For more details the reader is referred to Ref. [15].

The response functions with respect to the angle of attack are calculated using the CFD and grid motion approach for each sample conditions. In these simulations, the solution starts from a steady-state condition at angle of attack of $\alpha_{i}$ and Mach number of $M_{i}$, and then performing a small step in the angle of attack for all $t>0$. In these calculations, $M_{i}$ and $\alpha_{i}$ values correspond to the samples, and the side-slip angle is zero degrees at all times and the grid does not rotate at any time. The response functions are then computed by taking the differences between time-varying forces and moments occurring after the step and the steady-state solution at $\alpha=\alpha_{i}$ degrees, and dividing them by the magnitude of the step $(\Delta \alpha)$. For a weakly nonlinear system, the response will be nearly independent of the step magnitude (assuming that $\left.\alpha_{i}+\Delta \alpha \leq \alpha_{i+1}\right)$. The step value used in this study is a unit step. The lift and pitch moment indicial responses to a unit step change in the angle of attack from $\alpha_{i}=0$ are shown in Figs. 6(a) and (b) for Mach numbers of 0.1,0.3, and 0.5. These responses are in the linear regime and are used to create a linear ROM. The lift and pitch moment are plotted against the nondimensional time $s=2 V t / c$, where $V$ is the free-stream velocity, $t$ is the response

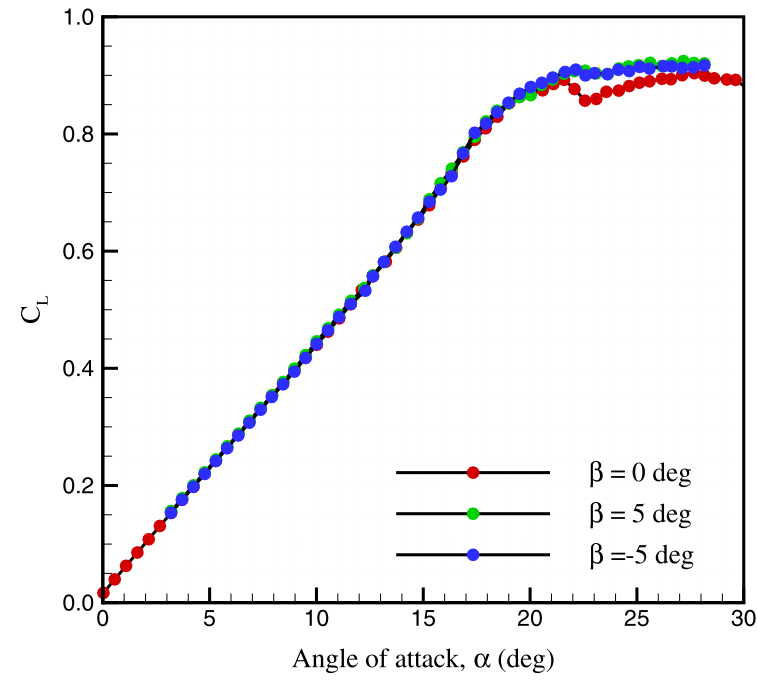

(a) Lift coefficient time, and $c$ is the reference length. Fig. 6(a) shows that the lift responses have a peak at $s=0$. Likewise, the pitch moment predicts a negative peak at this time as shown in Fig. 6(b). As the steady flow around the vehicle is disturbed by the grid motion, a compression wave and an expansion wave are formed on the lower and upper surface of the vehicle that cause a sharp peak in the responses [15]. As the response time progresses, the waves begin to move away from the vehicle, the lift starts to fall, and pitch moment starts to increase, and then responses asymptotically reach the steady-state values. Note that final time responses match with the slopes at zero angle of attack of static lift and pitch moment shown in Fig. 2. Figs. 6(a) and (b) also show that the initial peak becomes smaller for compressible flow. An explanation is given by Leishman [24]; this is due to the propagation of pressure disturbances at the speed of sound, compared to the incompressible case, where the disturbances propagate at infinite speed.

Figs. 6(c) and (d) show the lift and pitch moment responses at different angles of attack at Mach number of 0.3. These figures show that the initial values of responses are invariant with angle of attack, but the transient trend and steady state values change depending on the angle of attack. Not surprisingly, the lift and pitch moment responses at $\alpha=1^{\circ}$ and $\alpha=6^{\circ}$ match each other as shown in Fig. 6 . The lift and pitch moment are linear in this range of $\alpha$. At $\alpha=10^{\circ}$, there is a reduction in the lift and an increase in the pitch moment response. Formation of vortices at $\alpha=14^{\circ}$ causes the lift response to increase and pitch moment response to decrease. Significant changes can be seen in the responses at $\alpha=17^{\circ}$, where the outboard vortex has moved towards the wing apex. The responses at this angle have a long time transient solution before they reach the steady-state values. It should be noted again that final time responses at each $\alpha$ match with the slopes of static lift and pitch moment. For higher angles of attack, the grid motion approach could lead to a response instability as mentioned in Ref. [13].

The indicial responses in lateral loads due to a unit step change in the side-slip angle at different angles of attack are shown in Fig. 7 for Mach number of $0.1,0.3$, and 0.5 . In these simulations, the solution starts from a steady-state condition at zero degrees side-slip angle and an angle of attack of $\alpha_{i}$ at a Mach number of $M_{i}$, and then iterates such that the side-slip angle is held constant to one degree and the angle of attack is held constant to $\alpha_{i}$ for all $t>0$, where $M_{i}$ and $\alpha_{i}$ correspond to the samples. Likewise, for the lift and pitch moment, the initial peaks in lateral responses

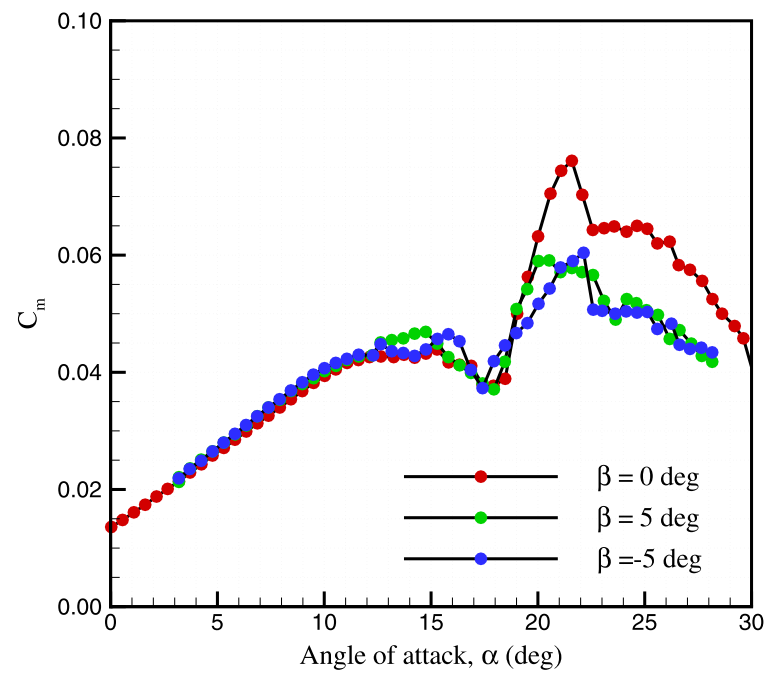

(b) Pitch moment coefficient

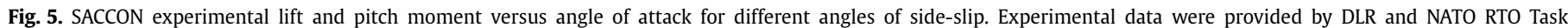
Group AVT-161. 


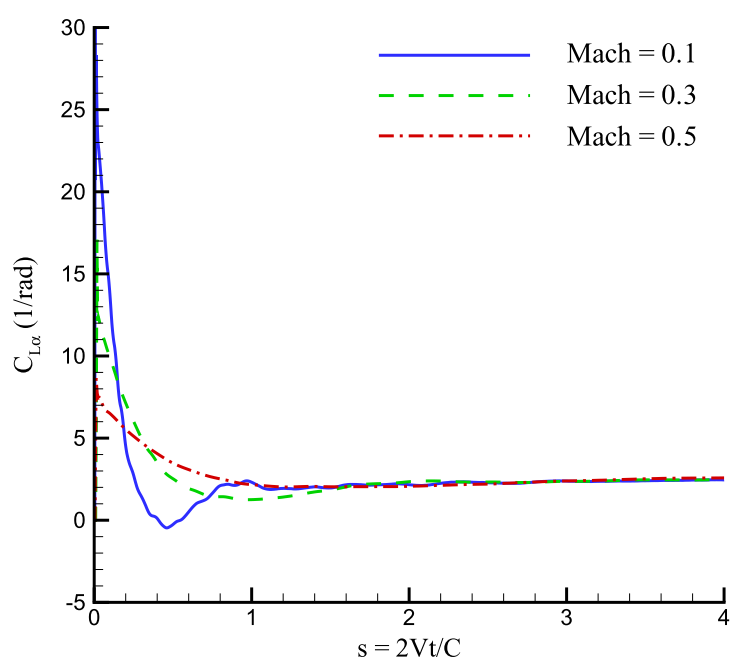

(a) linear $C_{L \alpha}$ functions

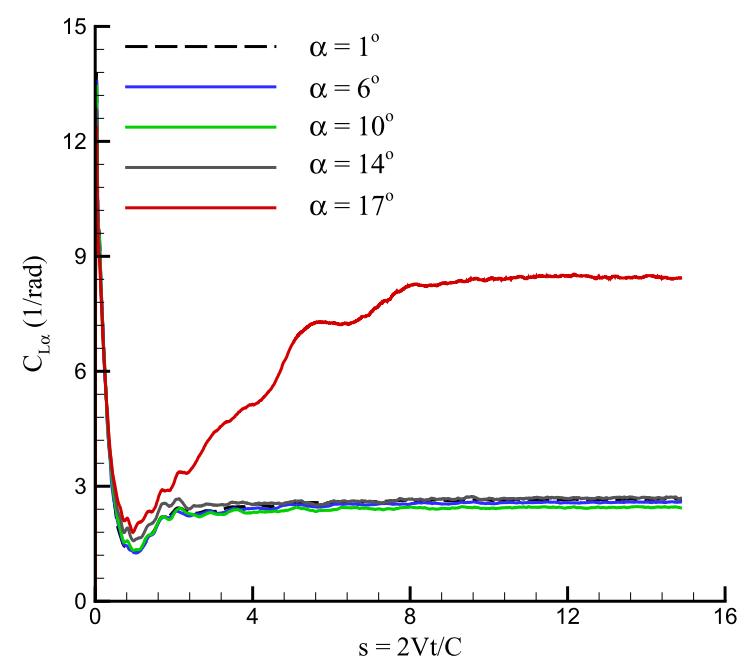

(c) nonlinear $C_{L \alpha}$ functions at $M=0.3$

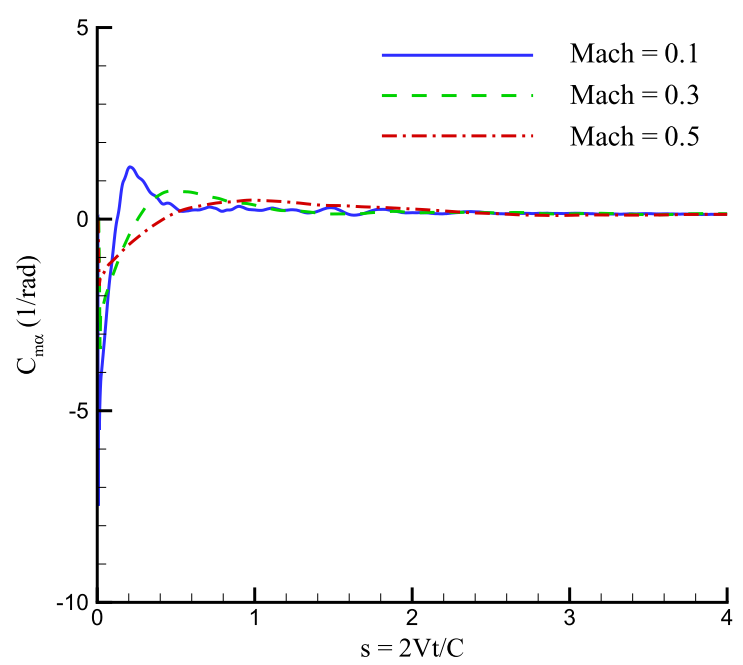

(b) linear $C_{m \alpha}$ functions

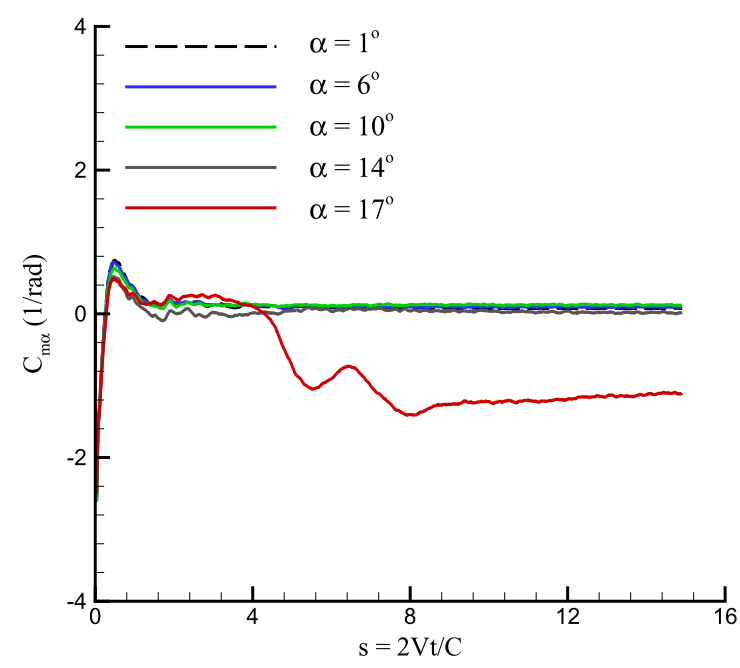

(d) nonlinear $C_{m \alpha}$ functions at $M=0.3$

Fig. 6. The lift and pitch moment response functions.

become smaller for compressible flow as shown in Figs. 7(a), (b), and (c). The responses in these figure correspond to $\alpha=0$ and used to create a linear ROM. Figs. 7(d), (e), and (f) show that lateral response functions vary nonlinearly with angle of attack. The sideforce responses for angles of attack below $10^{\circ}$ match each other, but large differences can be seen between responses at higher angles. Also, the yaw responses slightly change with the changes in the angle of attack for angles below $10^{\circ}$, and the nonlinear effects become increasingly important at higher angles. The roll moment responses do not match each other even at low angles of attack; this agrees with experimental measurements shown in Fig. 4. Fig. 7 shows that responses at $17^{\circ}$ have not been reached the steadystate solution at final simulation time.

Typically, the angle of attack effects are negligible for the responses due to the angular rates at low to moderate angles of attacks. Figs. 8(a)-(b) show the lift and pitch moment indicial responses respectively with a unit step change in pitch rate for Mach numbers of $0.1,0.3$, and 0.5 . Again there is an initial jump in lift as the grid starts to rotate, and the value decreases as Mach number increases. The lift response starts to fall a short time after initial excitation and then it reaches asymptotically a steady-state value, the so-called pitch dynamic derivative. Figs. 8(a)-(b) show that increasing Mach number results in the slight decrease of the lift and pitch damping derivatives. These calculations, along with a time-dependent surrogate model, were used to estimate the functions of $C_{L q}(t, M)$ and $C_{m q}(t, M)$ in the ROM equations. Also, the indicial functions with respect to roll and yaw rates are shown in Figs. 9-10, respectively. These calculations, along with a timedependent surrogate model, were used to estimate the functions of $C_{Y p}(t, M), C_{l p}(t, M), C_{n p}(t, M), C_{Y r}(t, M), C_{l r}(t, M)$, and $C_{n r}(t, M)$ in the ROM equations. The final time values of these functions are used to find dynamic derivatives as well. These derivatives are summarized in Table 3.

\subsection{Aircraft maneuvers}

The ROM equations were used for prediction of SACCON low and high angle-of-attack maneuvers. The low angle-of-attack maneuvers include barrel roll and Immelmann turn. The maneuvers were generated using DIDO to minimize final maneuver time subject to vehicle aerodynamics, mass properties, state and control constraints. The angle of attack is limited to $\left[-5^{\circ}, 10^{\circ}\right]$ while the maximum Mach number is 0.5 . The aircraft position and orientation during these maneuvers are shown in Fig. 11. In a barrel roll maneuver, the aircraft performs a complete rotation around its longitudinal axis. For the maneuver in this study, after a flight of $25 \mathrm{~s}$, SACCON's roll angle changes to $-360^{\circ}$ as shown in Fig. 12(d). For a barrel roll to the left, the maneuver is initiated by a pitch up 


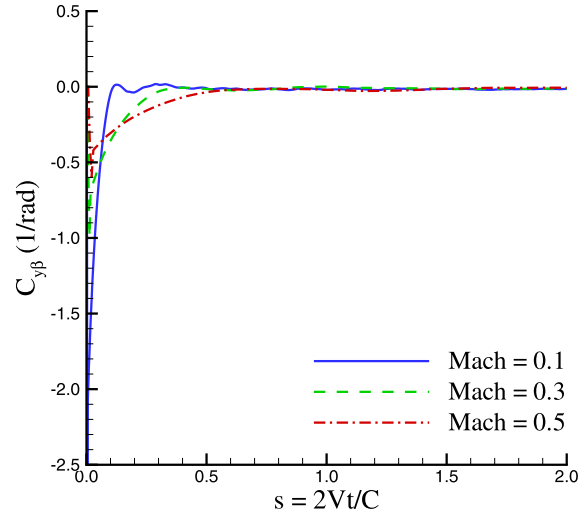

(a) linear $C_{Y \beta}$ functions

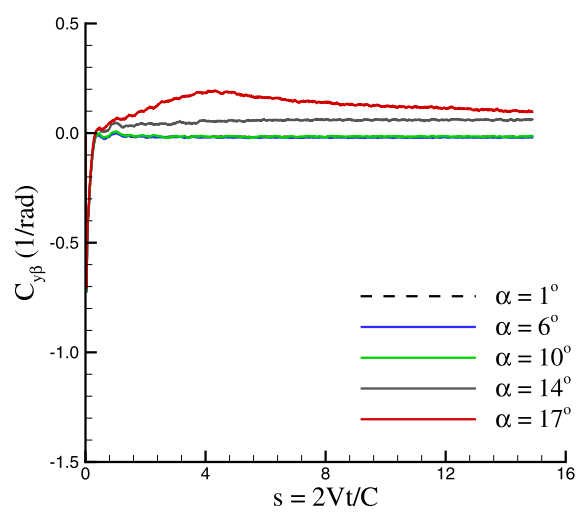

(d) nonlinear $C_{Y \beta}$ functions

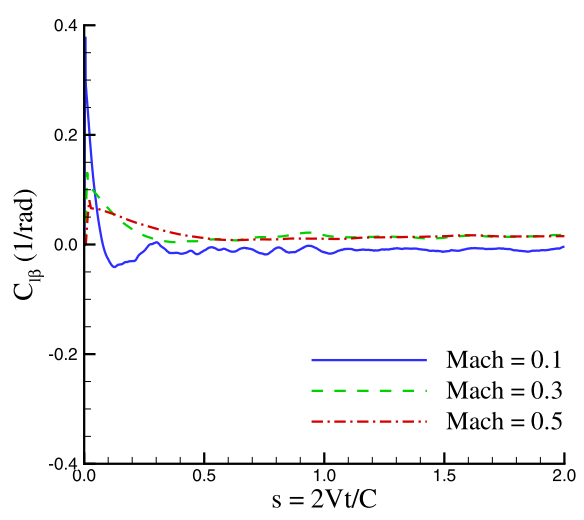

(b) linear $C_{l \beta}$ functions

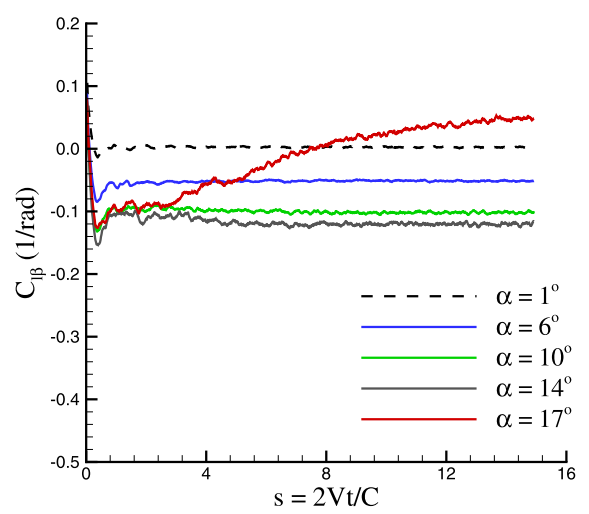

(e) nonlinear $C_{l \beta}$ functions

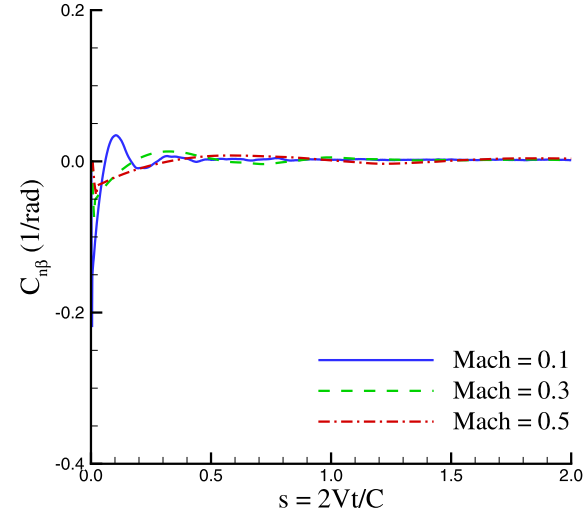

(c) linear $C_{n \beta}$ functions

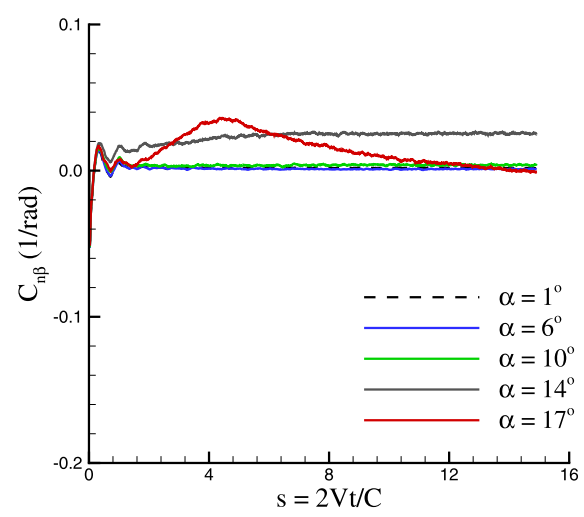

(f) nonlinear $C_{n \beta}$ functions

Fig. 7. The side-force, roll and yaw moments response functions. Nonlinear responses correspond to $M=0.3$.

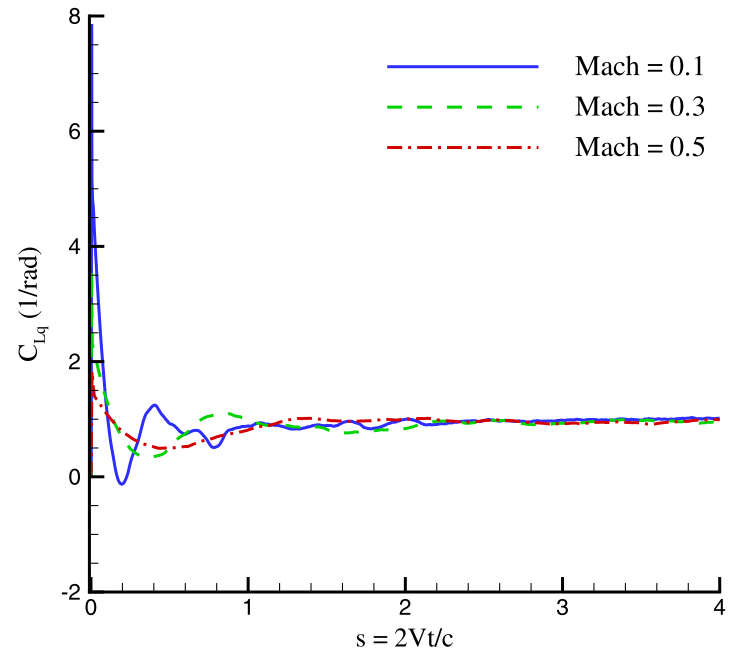

(a)

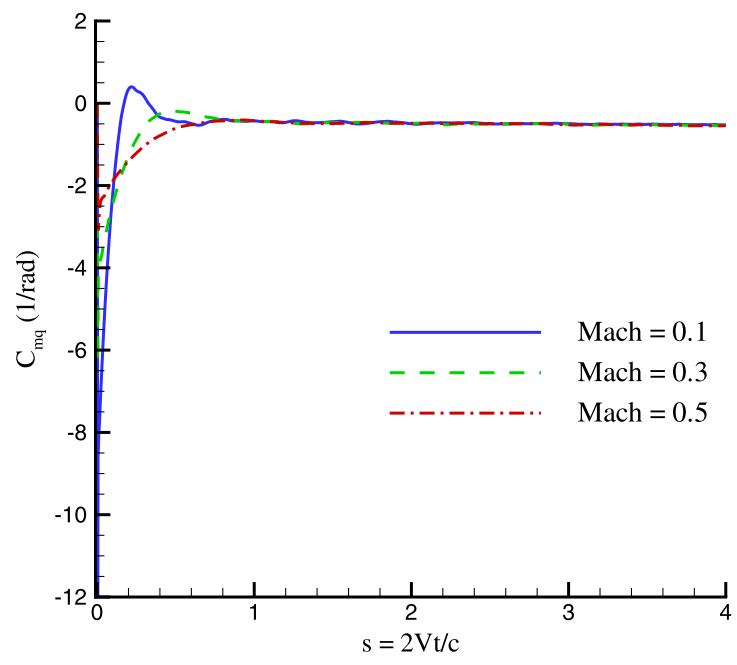

(b)

Fig. 8. The lift and pitch moment indicial functions with a unit step change of normalized pitch rate at different Mach numbers.

as well. During this part of the flight, the left wing is the lowest wing, while the aircraft yaws to the left as shown in Fig. 12(d). At the maximum altitude, the aircraft is nearly upside down. During the second half of the roll, the right wing is lowest and the aircraft tends to yaw to the right. The angle of attack range of this maneuver is $\left[-5^{\circ}, 5^{\circ}\right]$ as shown in Fig. $12(\mathrm{c})$. The altitude, velocity, side-slip angle, angular rates, and the time rates of change of $\alpha$ and $\beta$ are shown in Fig. 12. The angle-of-attack range of the Immelmann turn maneuver is $\left[-5^{\circ}, 10^{\circ}\right]$. The Immelmann turn as shown in Fig. 13 comprises a half loop with a half roll at the end. The maneuver starts with a steep climb and thus decreases the speed as shown in Fig. 13(b). At the maximum pitch angle, the aircraft heading suddenly changes from $0^{\circ}$ to $180^{\circ}$, which makes the aircraft final flight path exactly opposite of the initial path. As the heading starts to increase, the aircraft performs a half roll to level the wing as shown in Fig. 13(d). The final altitude is slightly higher than starting altitude as shown in Fig. 13(a). 


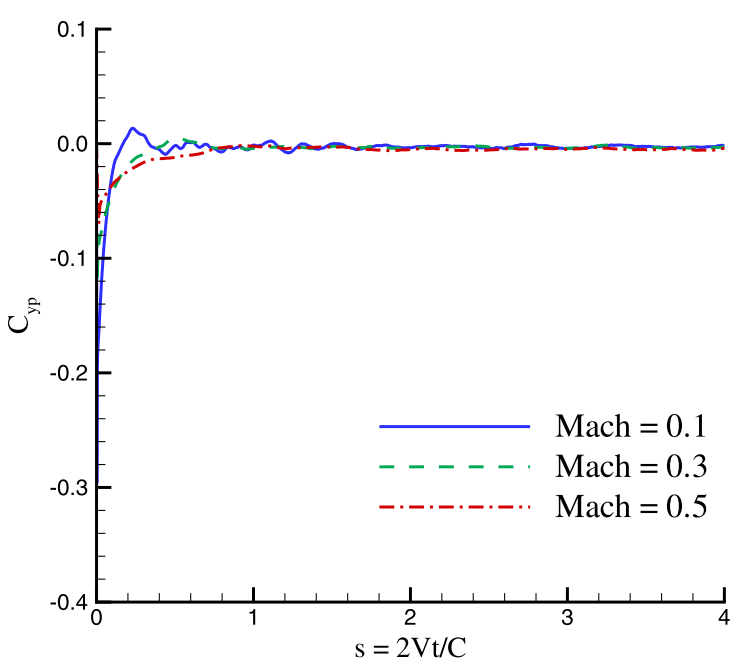

(a)

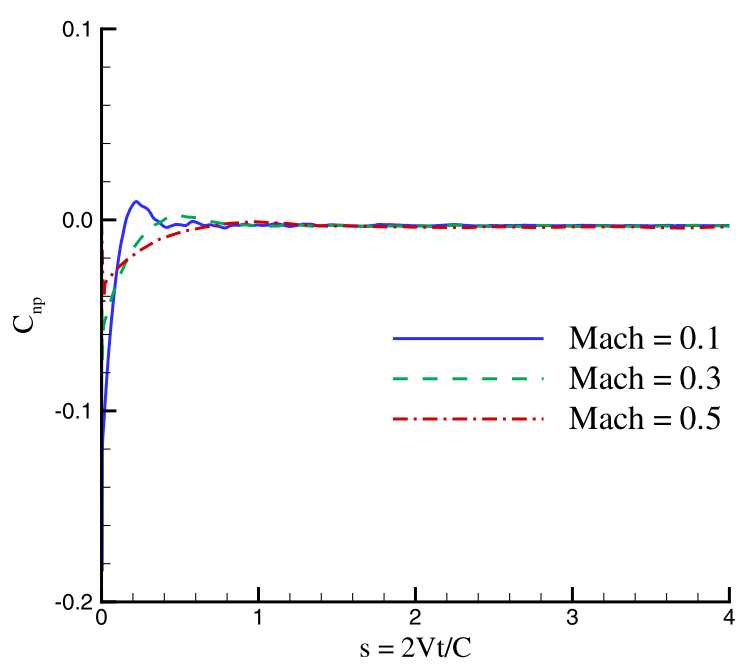

(c)

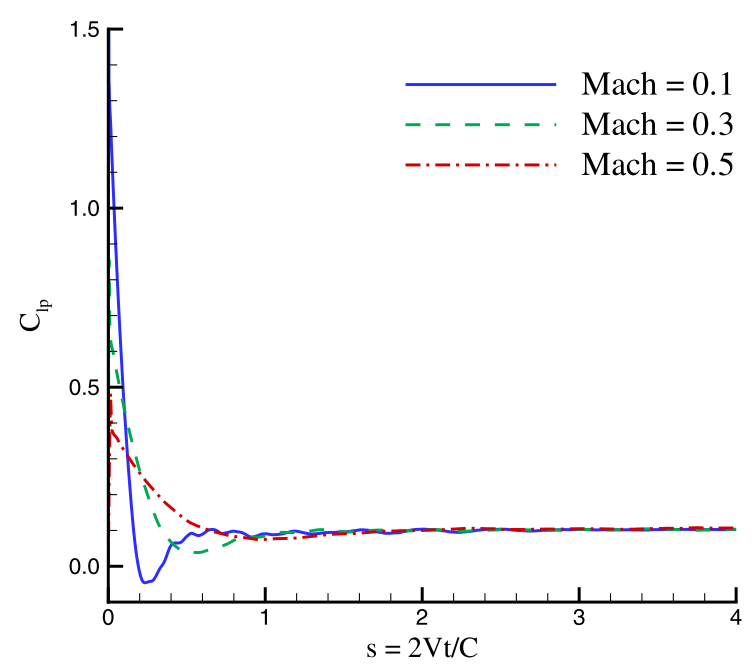

(b)

Fig. 9. The lateral indicial functions with a unit step change in the normalized roll rate.

The Immelmann turn maneuver is also considered for high angle-of-attack maneuvers. The maneuvers have trajectories similar to the low angle-of-attack Immelmann turn shown in Fig. 13 but the angle of attack and therefore $\dot{\alpha}$ have been increased. All other maneuver data including maneuver time are unchanged. Note that these new maneuvers are a purely theoretical example meant to evaluate the model at high angles of attack and are not meant to represent a physically achievable maneuver.

\subsection{ROM predictions}

The full-order model simulations of all maneuvers were calculated using URANS and grid motion. The full-order model and quasi-steady assumptions are labeled "CFD" and "Table" in the plots, respectively. The CFD computations started from a steadystate solution corresponding to the initial state of maneuver and then advanced in time using second-order accuracy. The motion files in Cobalt were generated from data values of each maneuver (angle of attack, Mach number, side-slip angle, and angular rates). These files define the rotations and displacements at discrete time instants and Cobalt then interpolates motion data using cubic-splines and moves the grid for each computational time step. The CFD solver reports time-dependent aerodynamic forces and moments in an inertia axis; these forces and moments were then transformed to the wind axis and normalized by the reference area and length and the dynamic pressure at each time step. Fig. 14 depicts the predicted aerodynamic loads of the barrel roll maneuver. The predictions from tables and a linear and nonlinear ROM are compared with CFD data. Note that the CPU time of the CFD simulation is around 67000 hours using 1024 processors. On the other hand, the model cost (calculation of static data and indicial functions) is made up-front and once the model is created the predictions take on the order of a few seconds. More details of the computational cost are given in the section on Computational cost Analysis. Figs. 14(a) and (b) show that lift and pitch moment predictions from all models match each other and with CFD solution fairly well. The angle of attack range of this maneuver is $\left[-5^{\circ}, 5^{\circ}\right]$ as shown in Fig. 12(c). At this range of $\alpha$ and the motion rates shown in Figs. 12(e) and (f), the unsteady and nonlinear effects into lift and pitch moment of this vehicle are small. Also, a very good match was found between model predictions and CFD data for side-force as shown in Fig. 14(c). The side-force is linear at low angles of attack and no significant unsteady effects are expected at these low angles. Figs. 14(d) and (e) show that predictions from nonlinear model and table match with CFD solution, but the linear model predictions are off because the yaw and roll moments are nonlinear with $\alpha$ changes. Nonlinear effects are more significant for roll moment. 


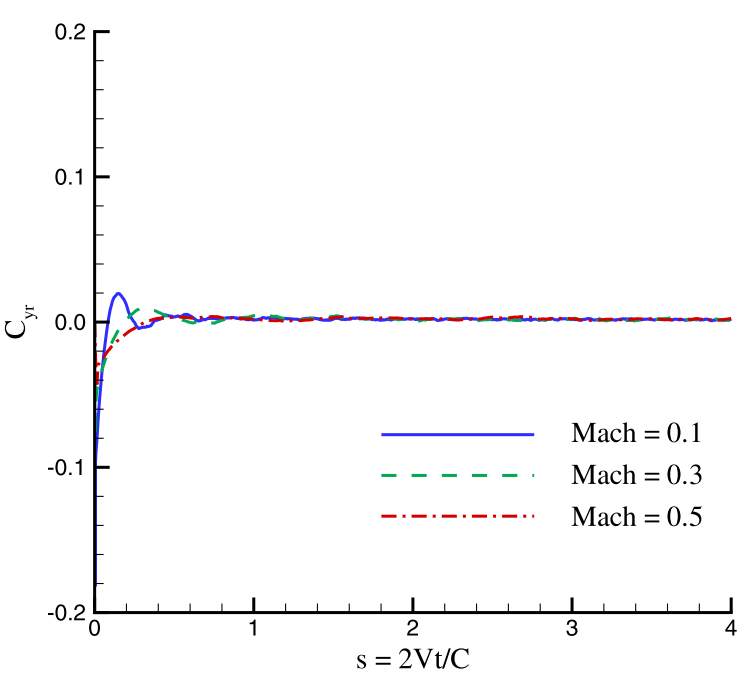

(a)

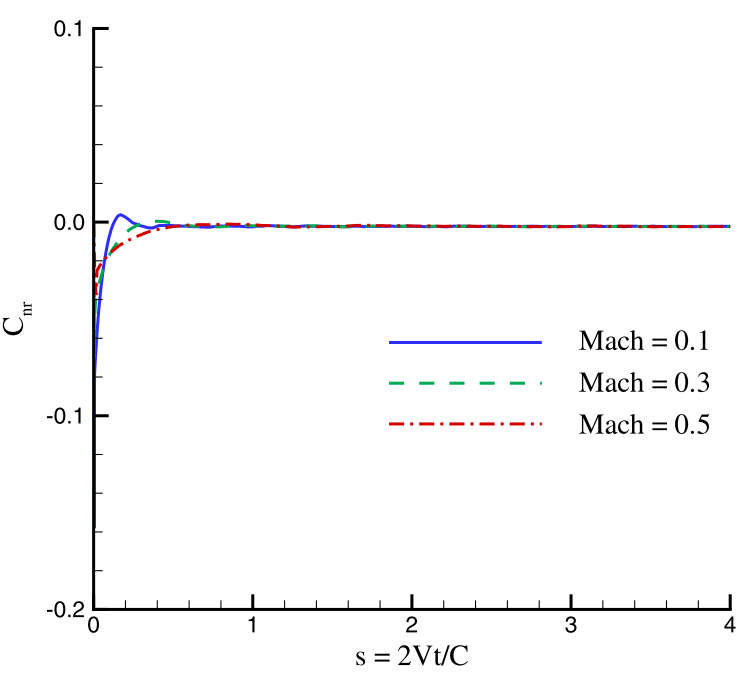

(c)

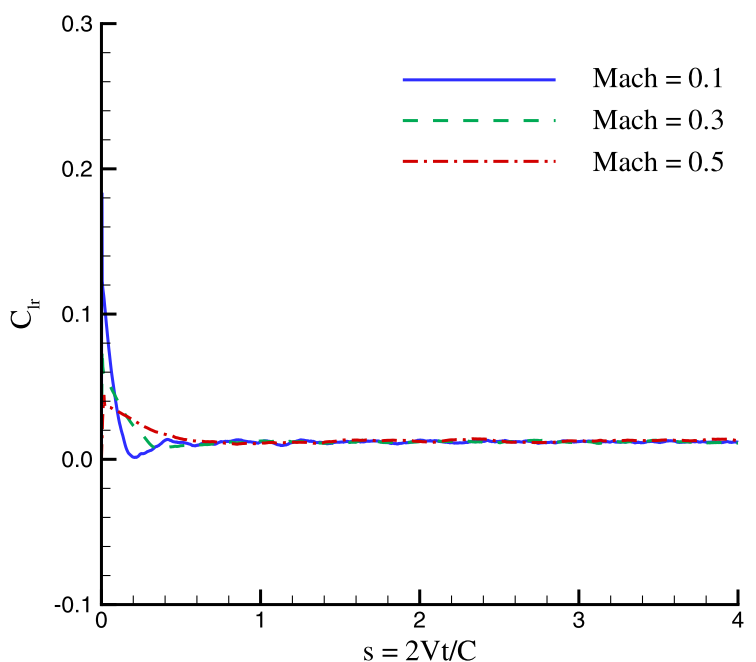

(b)

Fig. 10. The lateral indicial functions with a unit step change in the normalized yaw rate.

Table 3

Dynamic derivatives calculated from response functions at final time.

\begin{tabular}{lccc}
\hline Derivative $(1 / \mathrm{rad})$ & Mach $=0.1$ & Mach $=0.3$ & Mach $=0.5$ \\
\hline$C_{L q}$ & 1.021 & 0.969 & 0.988 \\
$C_{m q}$ & -0.526 & -0.537 & -0.546 \\
$C_{l p}$ & 0.1027 & 0.1035 & 0.1067 \\
$C_{n p}$ & -0.002956 & -0.003314 & -0.003770 \\
$C_{Y p}$ & -0.001550 & -0.003013 & -0.004231 \\
$C_{l r}$ & 0.01186 & 0.01118 & 0.01337 \\
$C_{n r}$ & -0.002179 & -0.002188 & -0.002365 \\
$C_{Y r}$ & 0.001760 & 0.001292 & 0.002015 \\
\hline
\end{tabular}

The CPU time of the CFD simulation of the Immelmann turn is around 70000 hours using 1024 processors. The comparisons between the new ROM with CFD data show good agreements in all coefficients as shown in Fig. 15. Fig. 15 also shows that table predictions match with the new model predictions. Table predictions are consistent with nonlinear and quasi-steady aerodynamics and they match CFD because no significant unsteady effects are expected at these low angles of attack and motion rates. However, the linear model predictions do not match, as shown in Fig. 15. The linear model overestimates the lift coefficient and underestimates pitch moment coefficient at large $\alpha$ values. Also, the predictions from a linear model do not match with roll and yaw moment co- efficients from CFD, since the model formulation is valid only in linear regimes. These results show the limits of a linear model to accurately predict aerodynamics of a maneuvering SACCON.

An Immelmann turn maneuver with an angle of attack in the range of $\left[-5^{\circ}, 15^{\circ}\right]$ is considered next. The angle of attack variation during the maneuver is shown in Fig. 16(a). All other maneuver data including maneuver time are given in Fig. 13. Since the maneuver time is fixed but angle of attack is increased, the new maneuver has higher values of $\dot{\alpha}$ compared with low angle-ofattack Immelmann turn. Note again that this maneuver is a purely theoretical example meant to evaluate the model at high angles of attack and $\dot{\alpha}$ and is not meant to represent a physically achiev- 


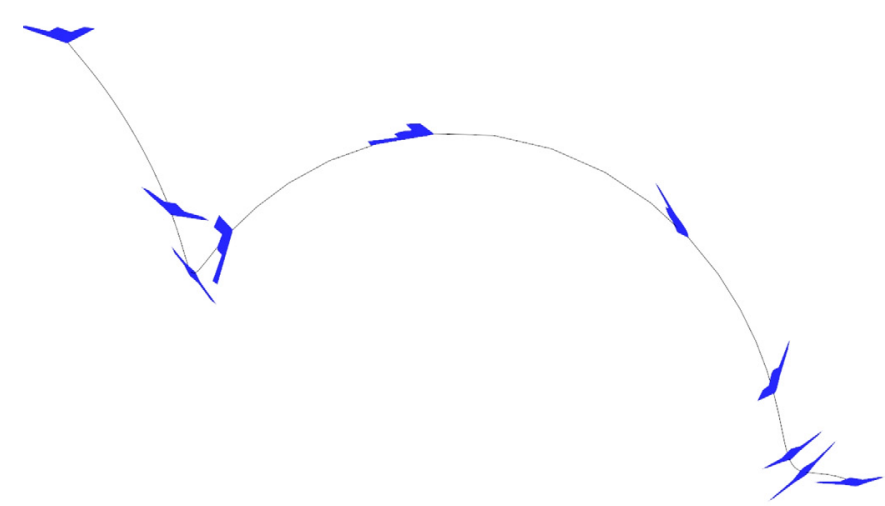

(a) Barrel roll

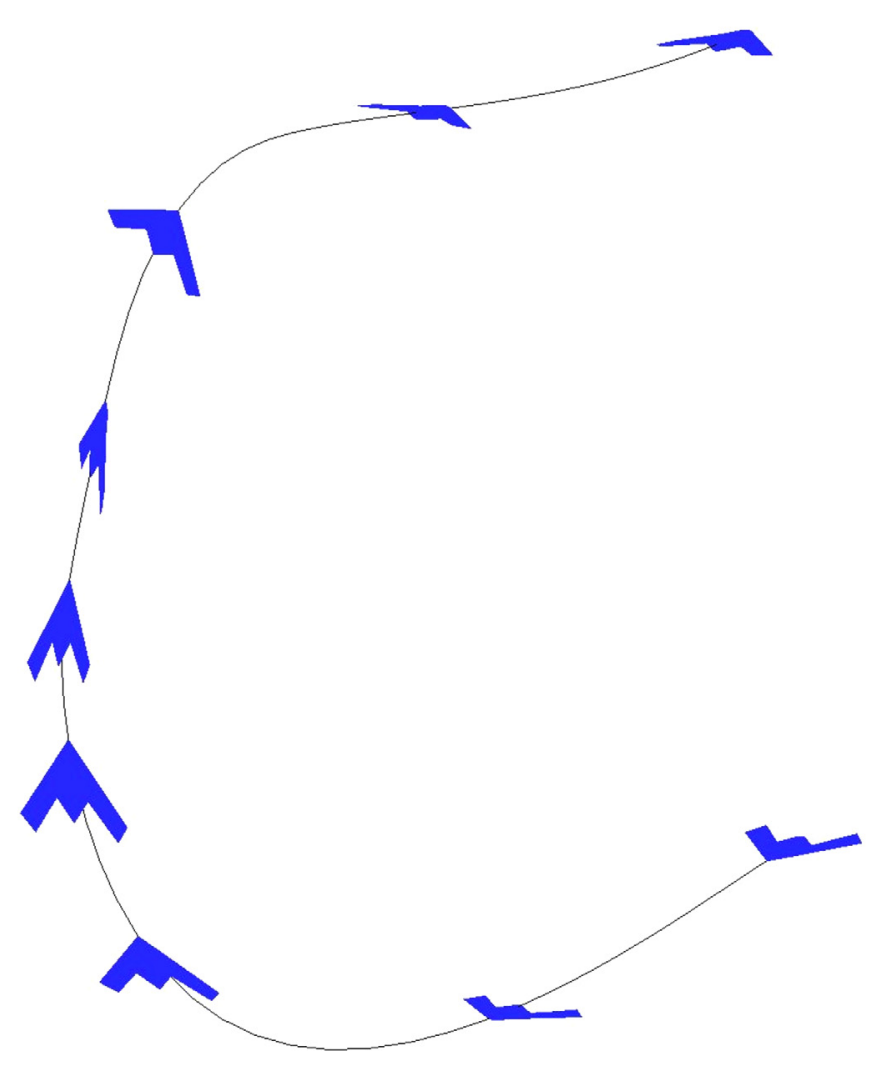

(b) Immelmann turn

Fig. 11. Flight trajectories.

able maneuver. In Fig. 16, CFD data are compared with ROM and table predictions. The results show that ROM predictions match CFD data quite well. However, table predictions do not match lift and pitch-moment coefficients; they overestimate lift and underestimate pitch moment. Differences are significant at high angles of attack, where the nonlinear vortex effects take place. There are significant history effects in lift and pitch moment due to high rate of angle of attack changes in presence of these vortices; tables cannot predict these history effects.

Next, an Immelmann turn maneuver with an angle of attack in range of $\left[-5^{\circ}, 17^{\circ}\right]$ is considered. The angle of attack variation during maneuver is shown in Fig. 17(a) and other maneuver data are given in Fig. 13. CFD data are compared with ROM and table predictions in Fig. 17. The results show that although ROM performs better than tables in predicting lift and pitch moment, but it does not accurately predict the CFD data as well. The dis- crepancies are mainly due to assumptions made and challenges in calculation of indicial functions at high angles of attack. The lateral coefficients become increasingly nonlinear with side-slip angle for $\alpha$ above $15^{\circ}$. The indicial responses due to angular rates also become angle-of-attack dependent at these conditions. The results also showed that lateral indicial functions did not reach the steady-state solutions at the final time of response simulation. To improve the model, it is suggested to include the side-slip angle in the input space. However, this requires a better sampling method that reduces the number of CFD calculations. Also, the indicial functions with respect to $p, q, r$ need to include an angle of attack dependency. A way to calculate high angle-of-attack indicial functions in CFD is to approximate the functions from oscillation motions. However, the functions found this way are highly dependent on the amplitude and frequency of motions. A modified model based on indicial functions, for example Beddoes and Leishman [25], could also be used for aerodynamics modeling at high angles of attack.

\subsection{Computational cost analysis}

Table 4 compares the costs to build the ROMs and of running the CFD model. The linear and nonlinear models required about (around) 19000 and 466000 hours of CPU time, respectively. However, these costs are up-front and once the models are created they could be used for aerodynamics prediction of a wide range of aircraft maneuvers. The CFD simulation of aircraft maneuvers is computationally very expensive and time consuming; for example, an Immelmann turn maneuver took around 70000 hours of CPU to run. On the other hand, the ROM predictions took on the order of a few seconds after spending an initial cost in building the model.

\section{Conclusions}

This paper investigates the use of ROMs that significantly reduce the CFD simulation time required to create a full aerodynamics database, and improve the accuracy of prediction of aircraft static and dynamic characteristics over quasi-steady predictions. The ROM considered was based on linear and nonlinear indicial response methods. The response functions consist of aircraft responses to step changes in the angle of attack, pitch rate, side-slip angle, roll, and yaw rates. All these functions were calculated using direct response simulation in URANS with the aid of rigid grid motion tool. A time-dependent surrogate model was described to find the response functions dependency on the angles of attack and Mach numbers.

The test case used was the SACCON UCAV scaled up to fit the characteristics of a full size aircraft. Time-optimal maneuvers were generated using the DIDO code to minimize final maneuver time subject to vehicle aerodynamics, mass properties, state and control constraints. The comparison between unsteady simulation of maneuvers with ROM predictions showed the consistency of predictions for all coefficients for angles of attack below $15^{\circ}$. The linear model predictions do not match with CFD for yaw and roll moment coefficients even at low angles of attack. Also, table predictions, which are consistent with quasi-steady aerodynamics, do not match with the CFD for maneuvers at high angles of attack and high rate of angle of attack changes. The results showed the limits of ROM for predicting CFD data at higher angles of attack due to assumptions made in the model and difficulties in calculation of indicial functions at high angles of attack.

\section{Conflict of interest statement}

We wish to confirm that there are no known conflicts of interest associated with this publication and there has been no signifi- 


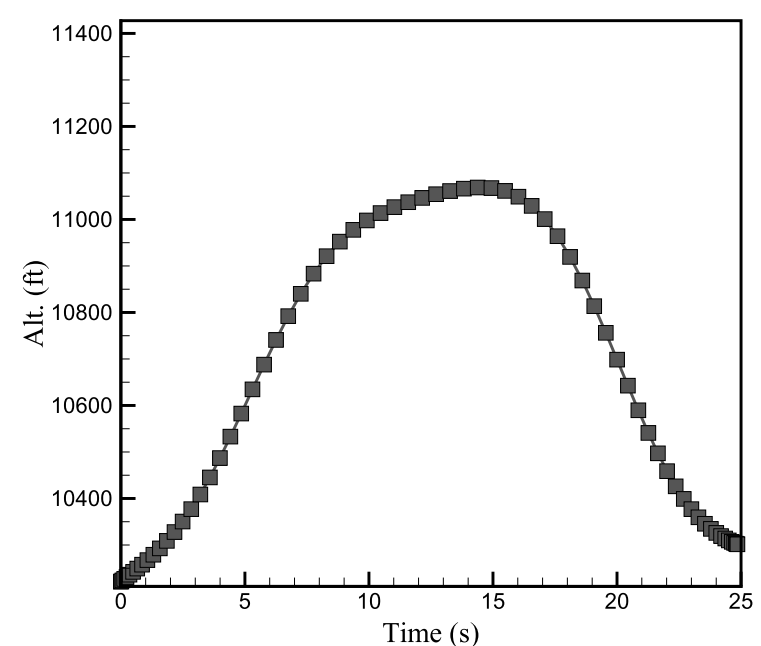

(a) flight altitude

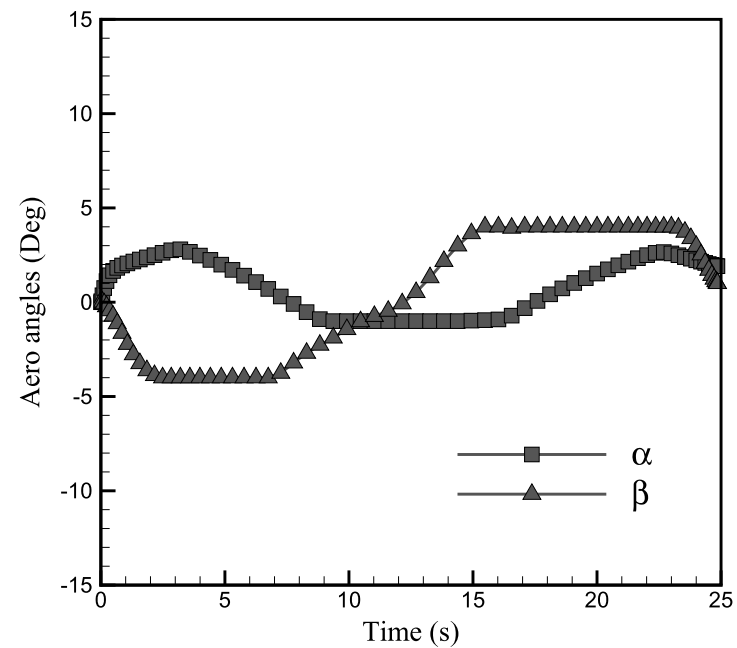

(c) aero angles

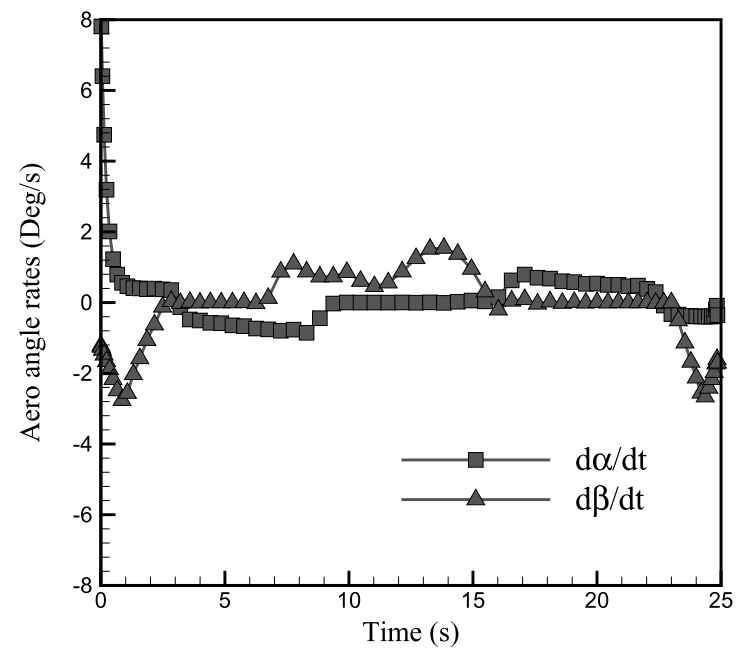

(e) aero angle rates

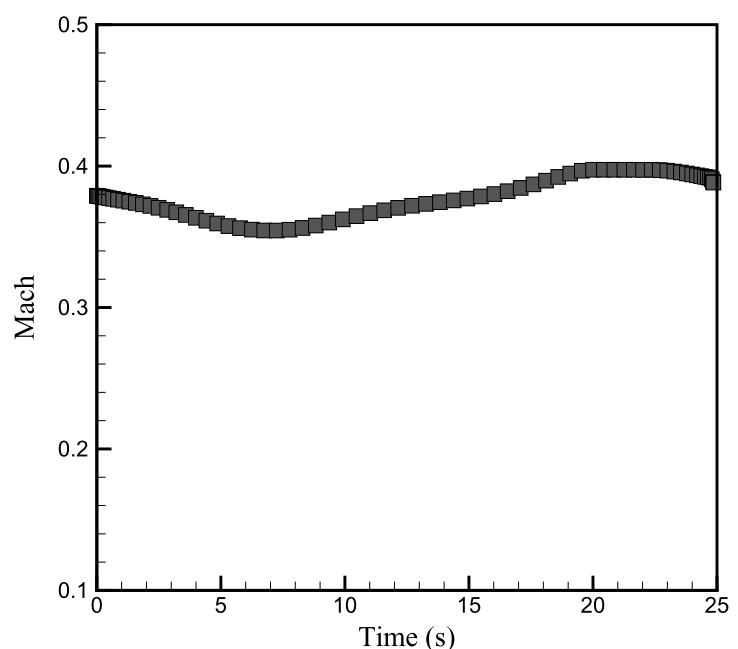

(b) Mach number

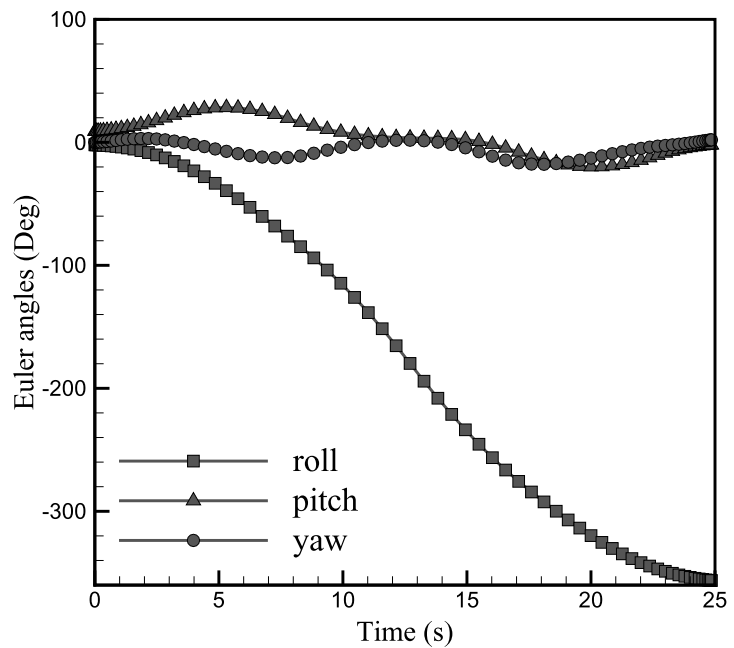

(d) euler angles

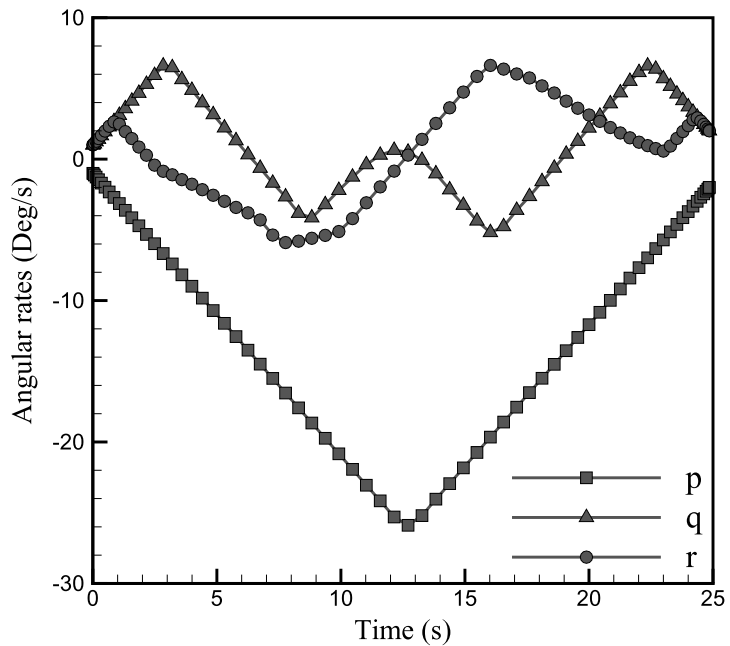

(f) angular rates

Fig. 12. Barrel roll maneuver. 


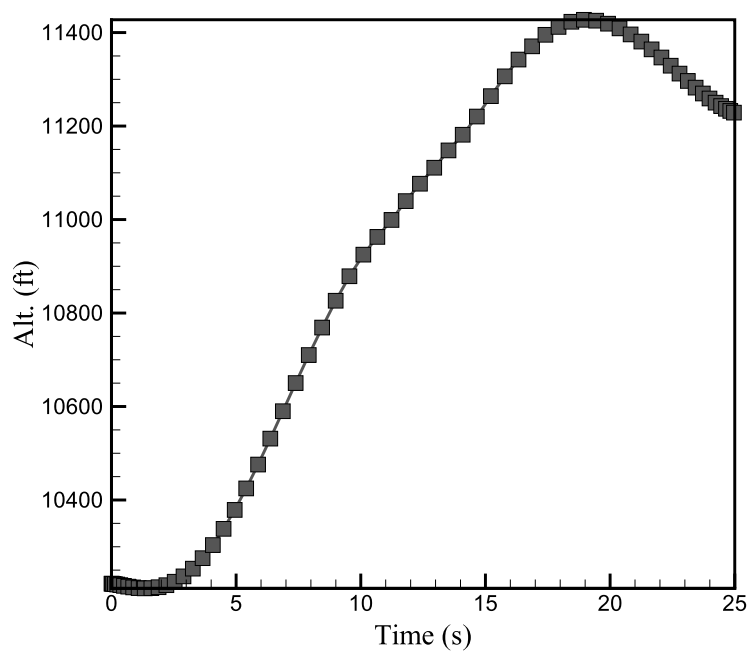

(a) flight altitude

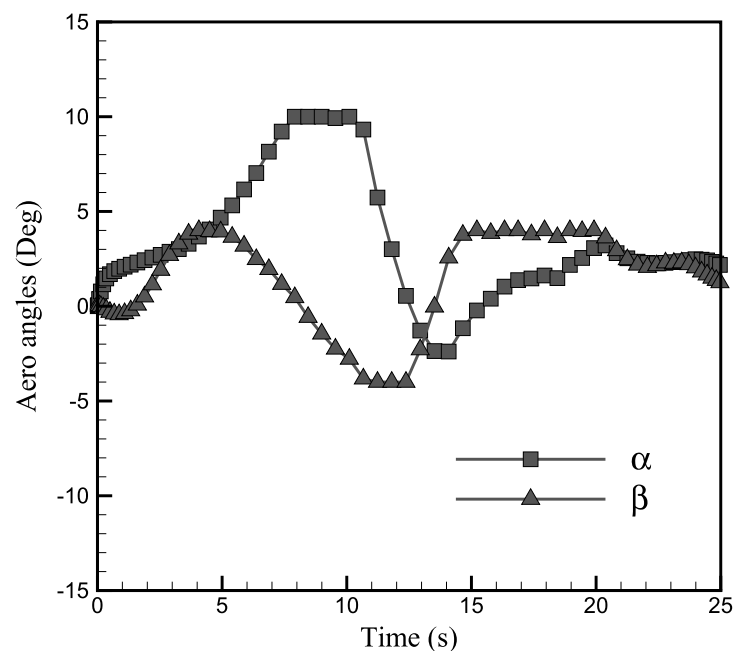

(c) aero angles

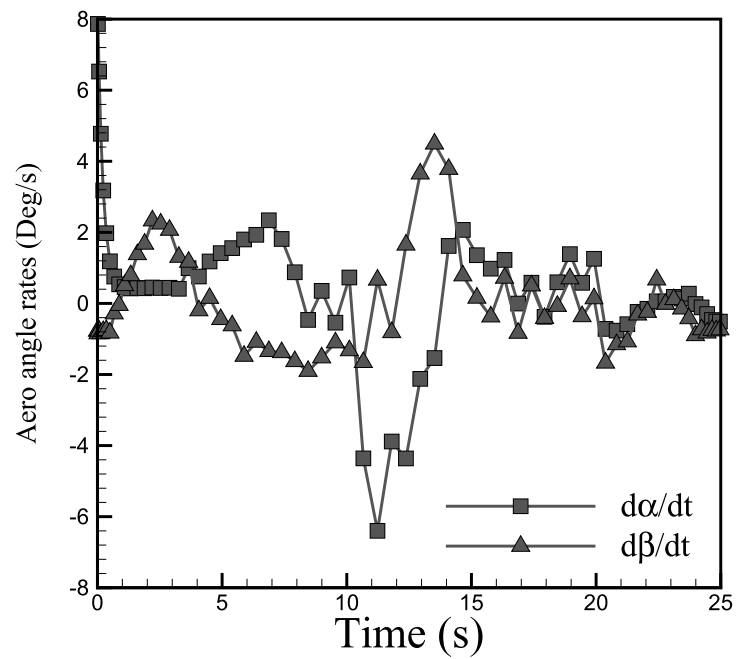

(e) aero angle rates

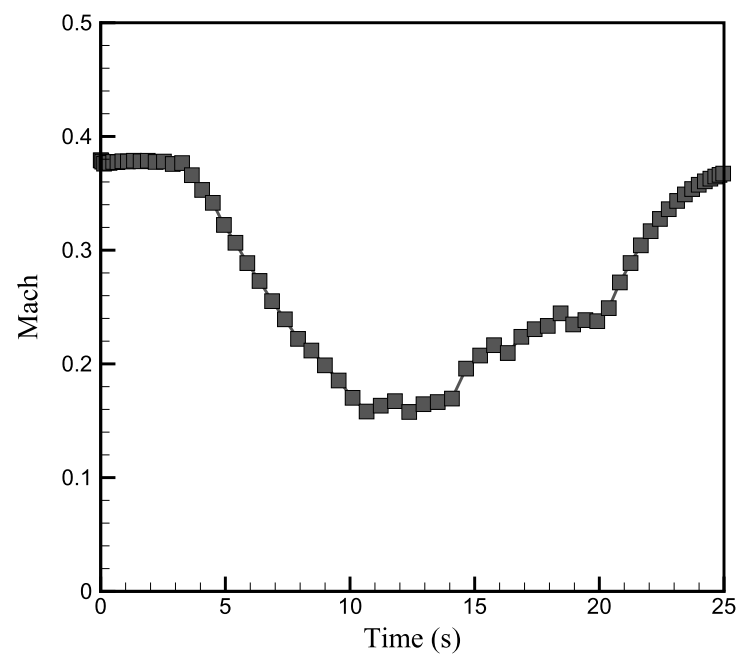

(b) Mach number

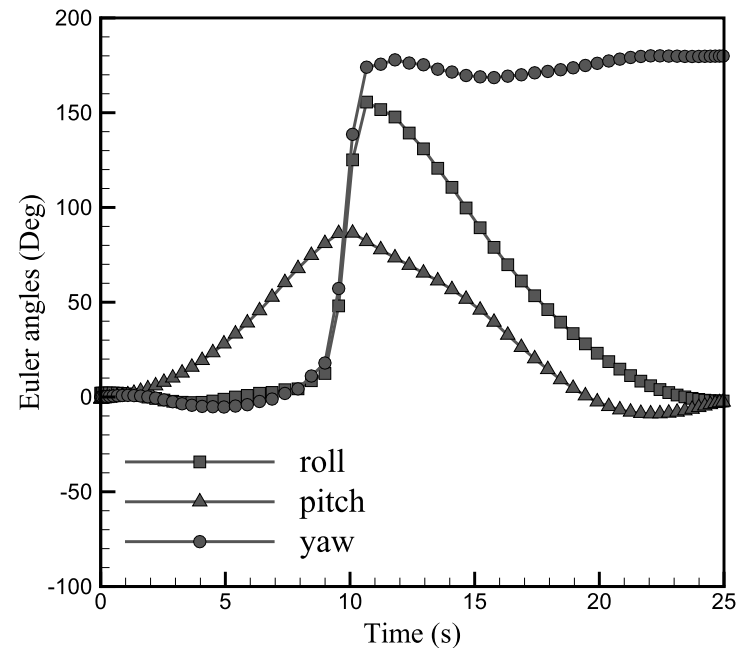

(d) euler angles

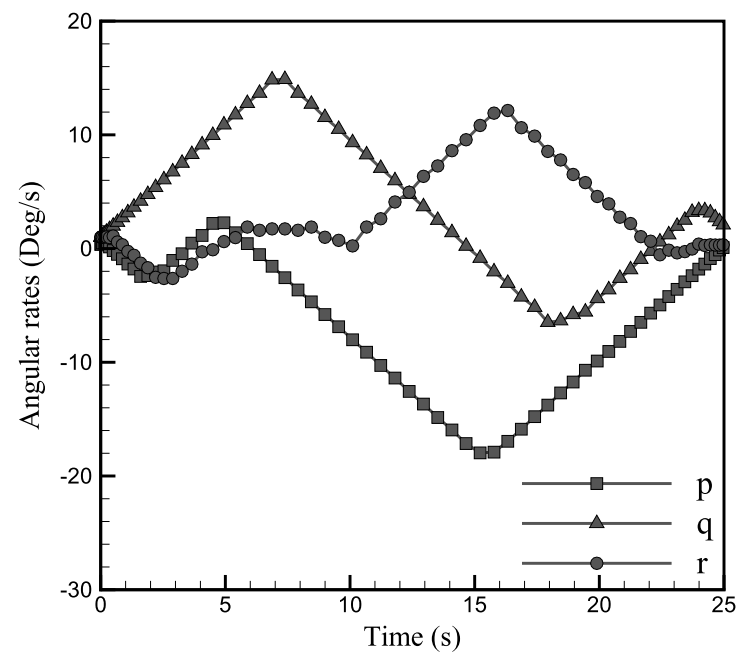

(f) angular rates

Fig. 13. Immelmann turn maneuver. 


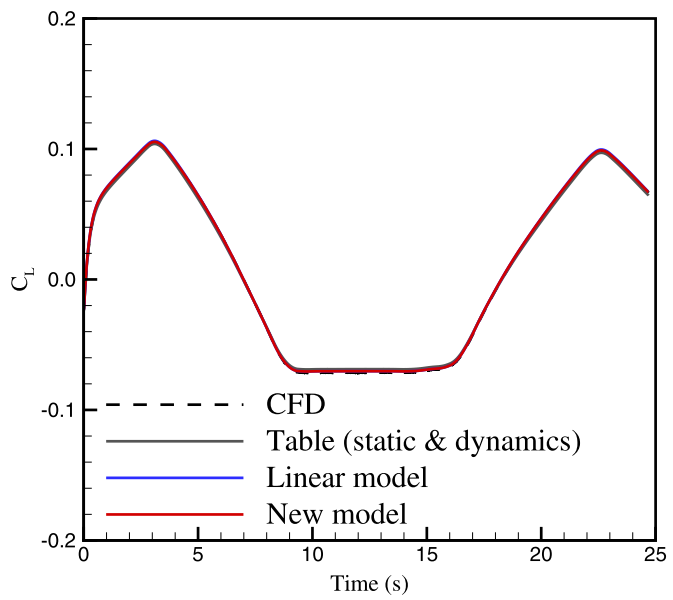

(a) Lift coefficient

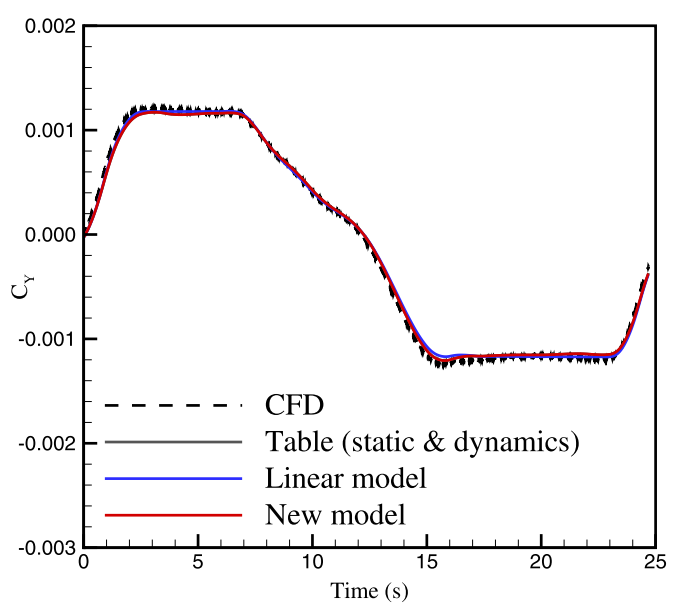

(c) Side-force coefficient

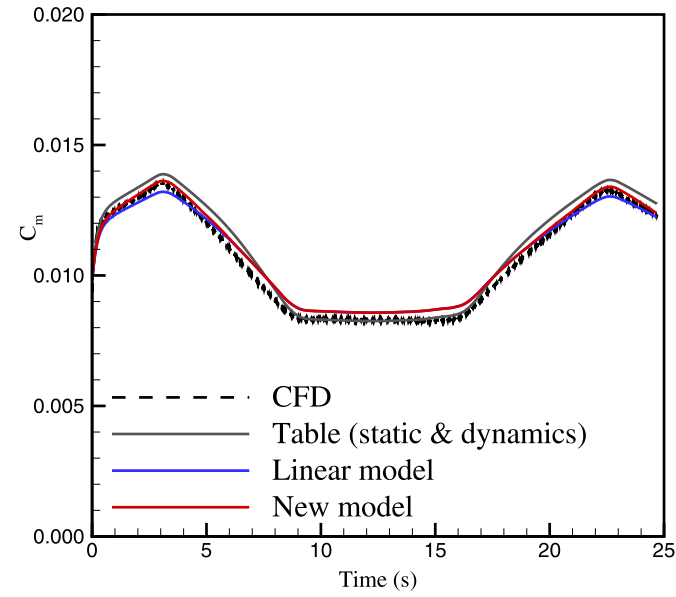

(b) Pitch moment coefficient

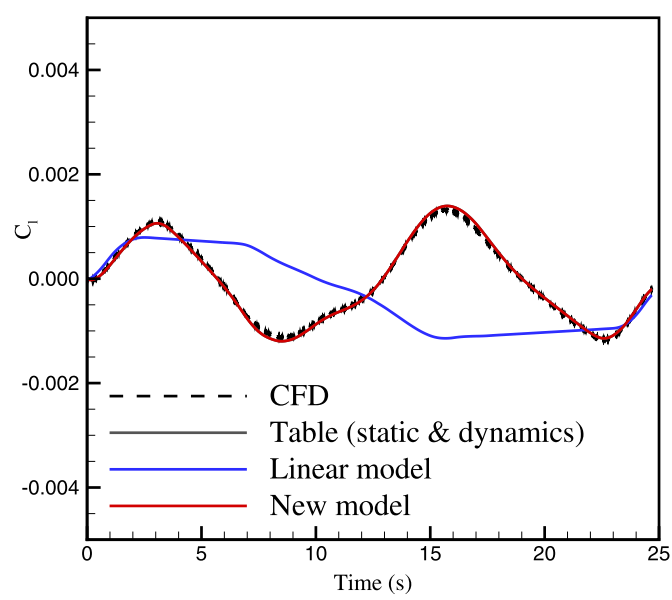

(d) Roll moment coefficient

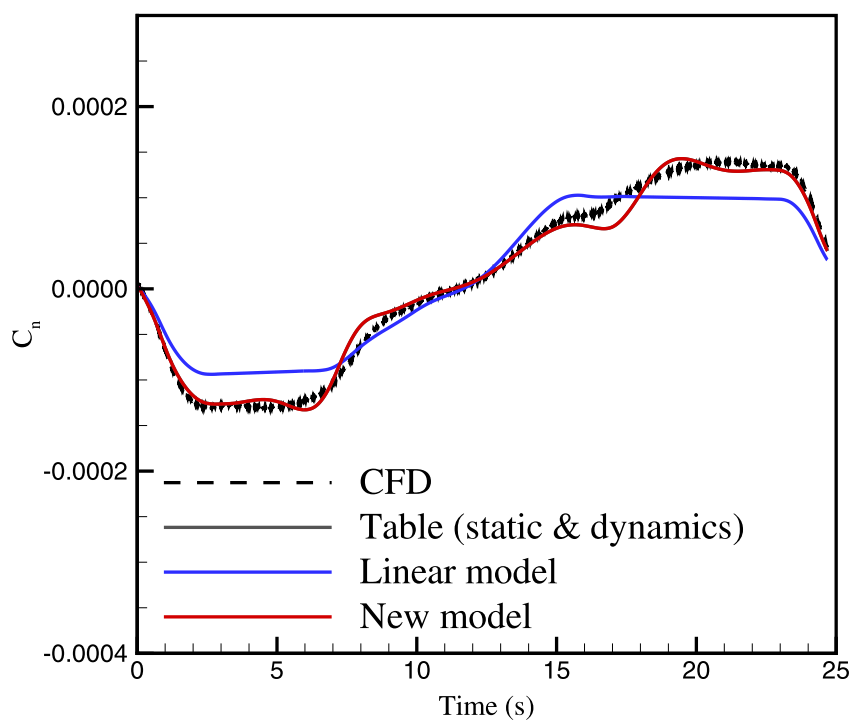

(e) Yaw moment coefficient

Fig. 14. Aerodynamic modeling of barrel roll maneuver. Table predictions are consistent with quasi-steady aerodynamics. 


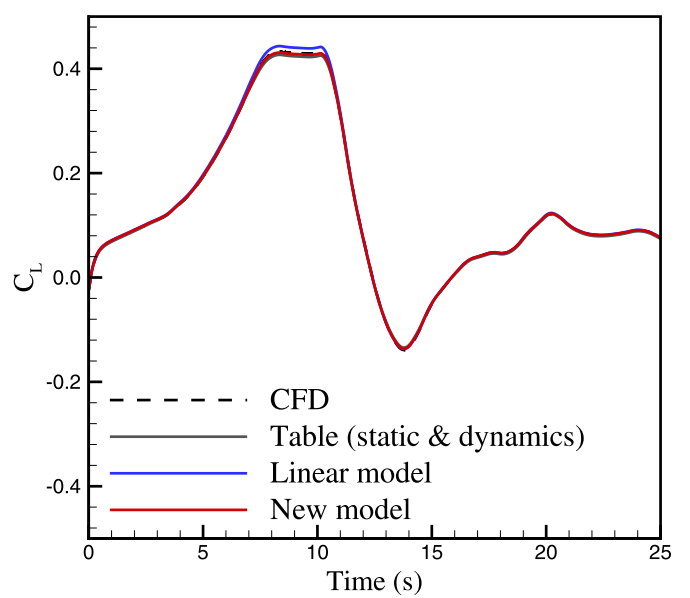

(a) Lift coefficient

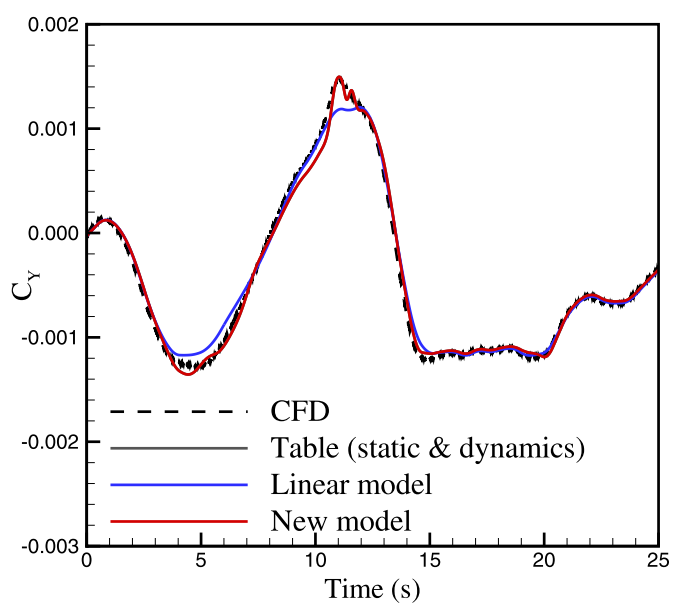

(c) Side-force coefficient

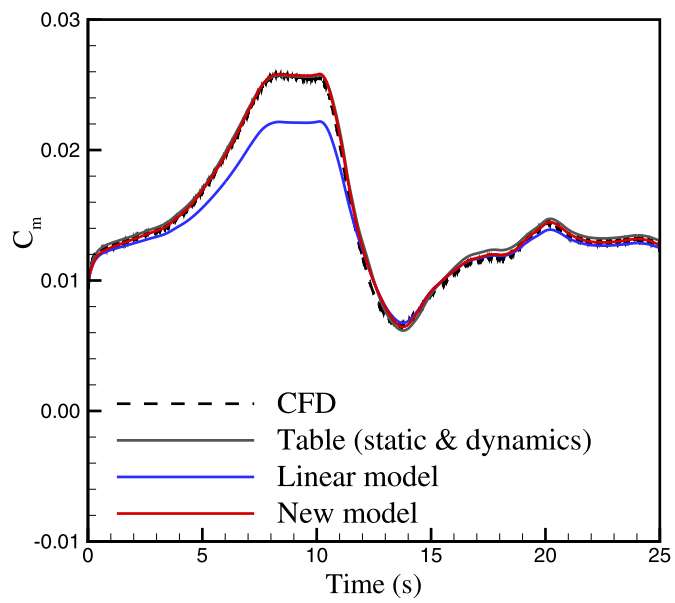

(b) Pitch moment coefficient

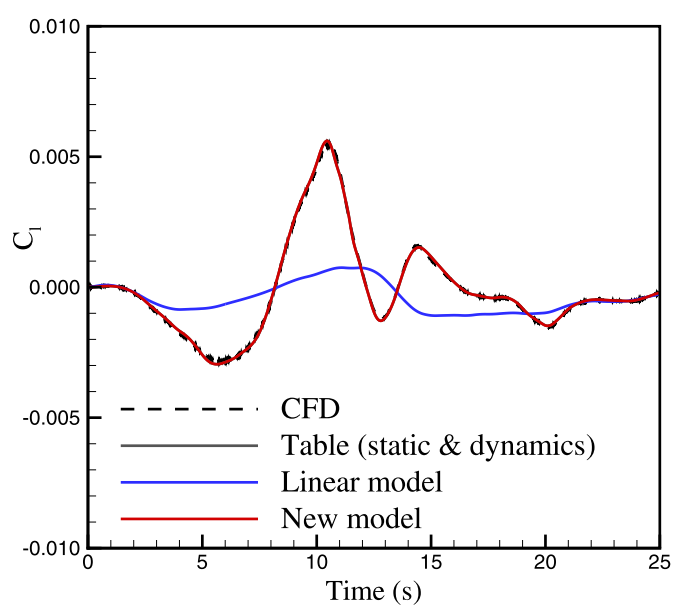

(d) Roll moment coefficient

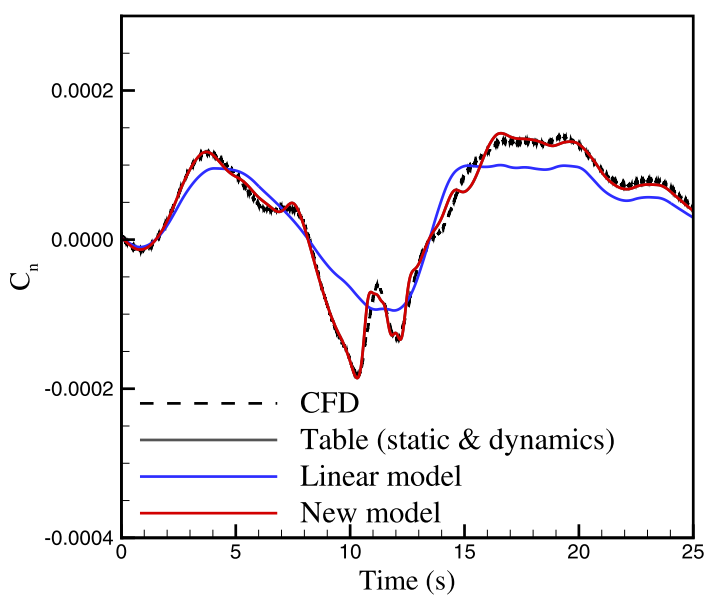

(e) Yaw moment coefficient

Fig. 15. Aerodynamic modeling of Immelmann turn maneuver. 


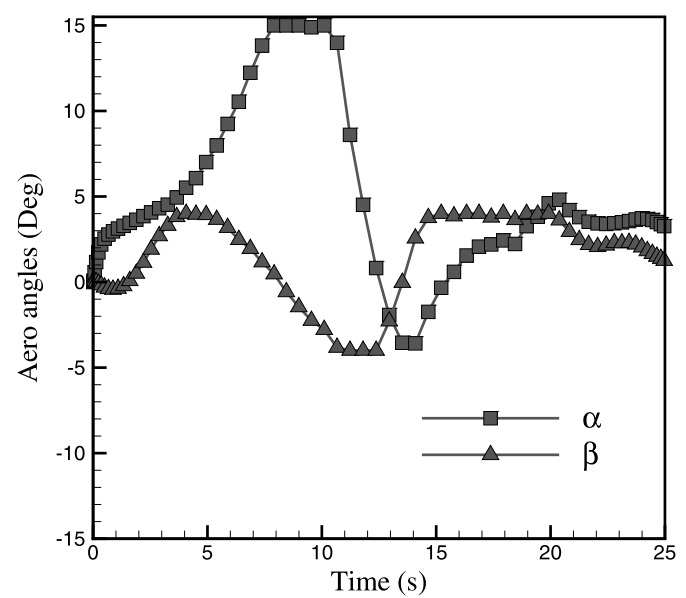

(a) Aero angles

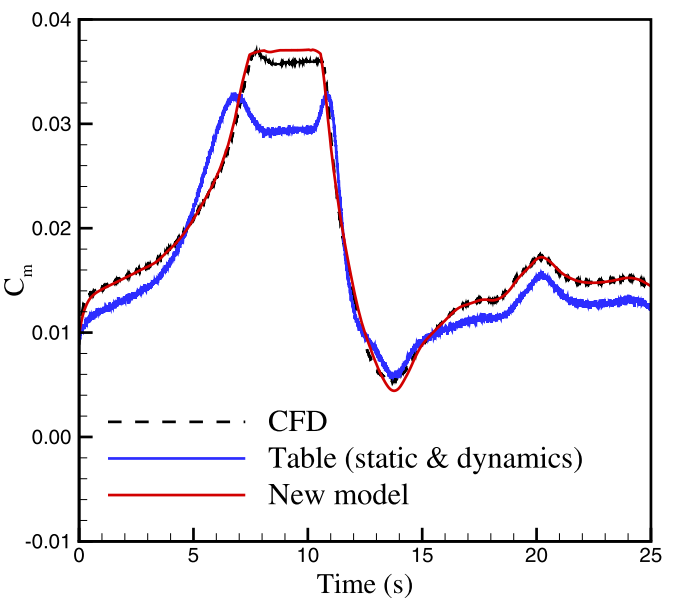

(c) Pitch moment coefficient

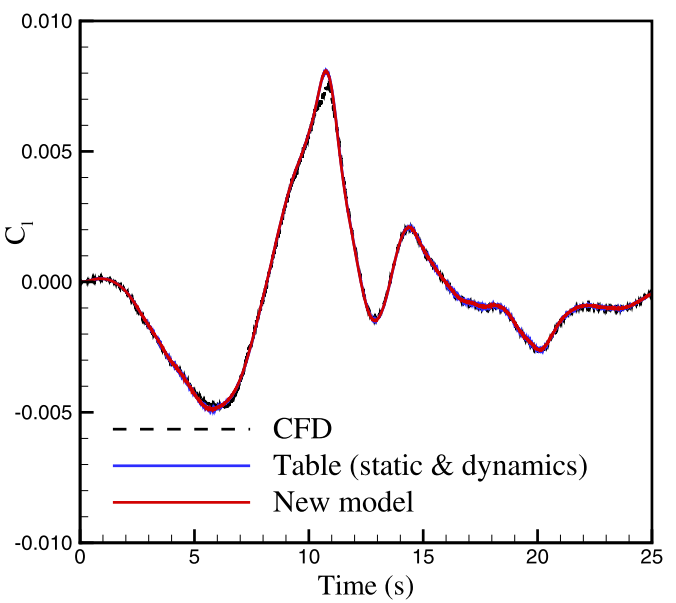

(e) Roll moment coefficient

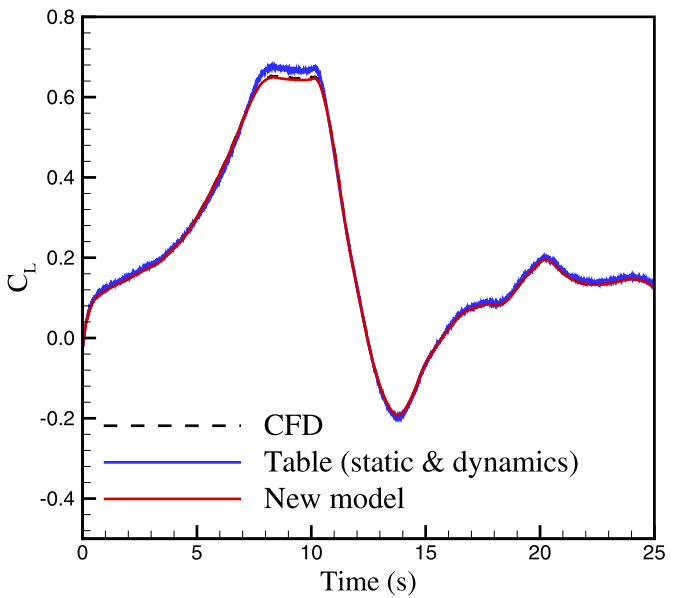

(b) Lift coefficient

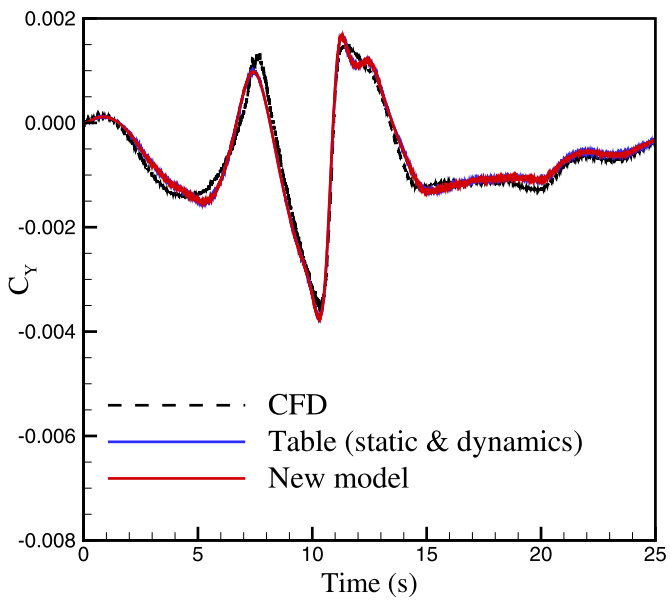

(d) Side-force coefficient

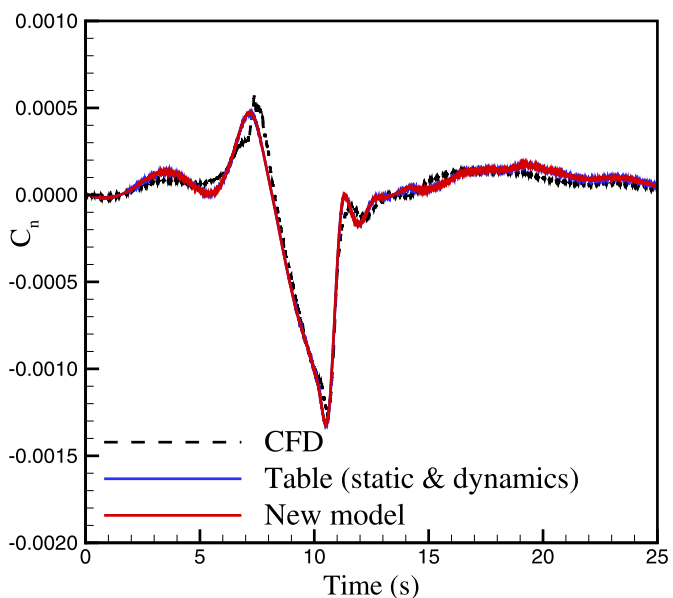

(f) Yaw moment coefficient

Fig. 16. Aerodynamic modeling of Immelmann turn maneuver for angles of attack up to $15^{\circ}$. 


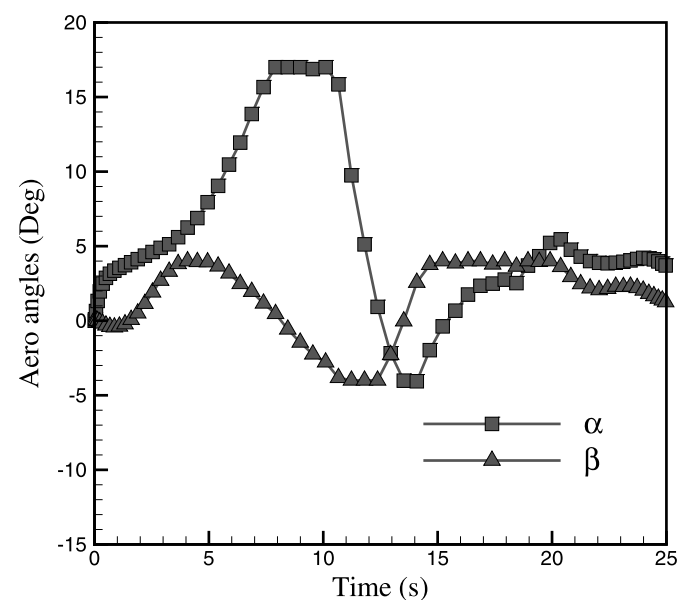

(a) Aero angles

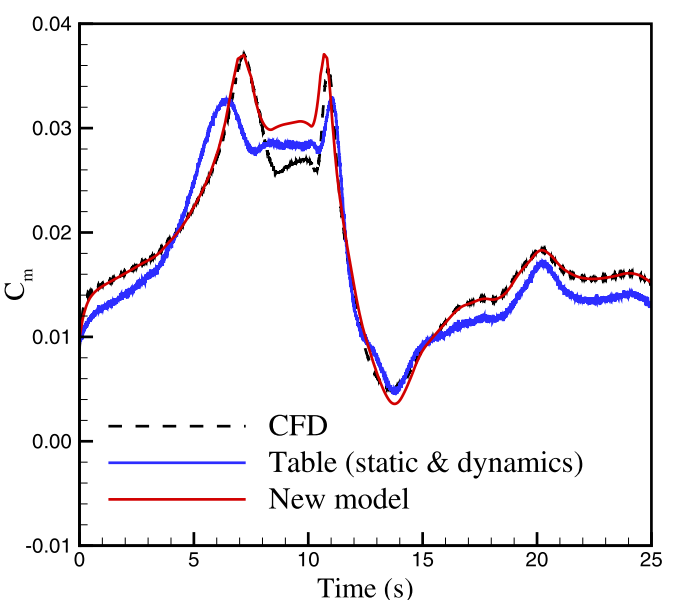

(c) Pitch moment coefficient

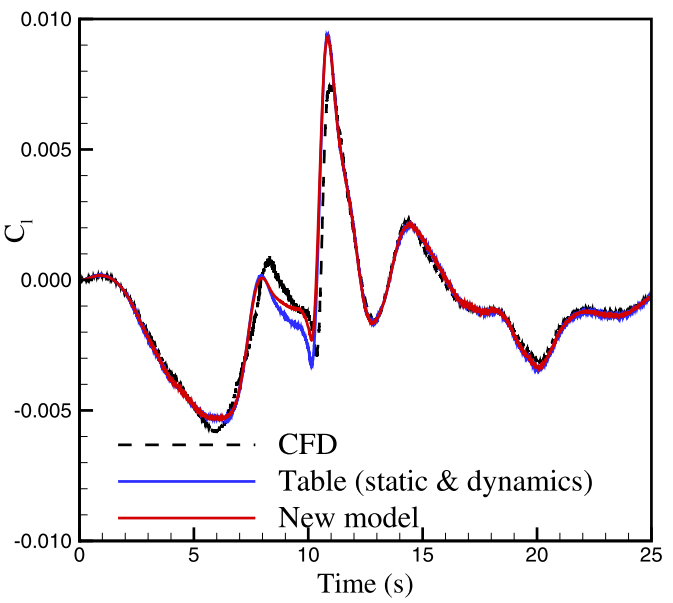

(e) Roll moment coefficient

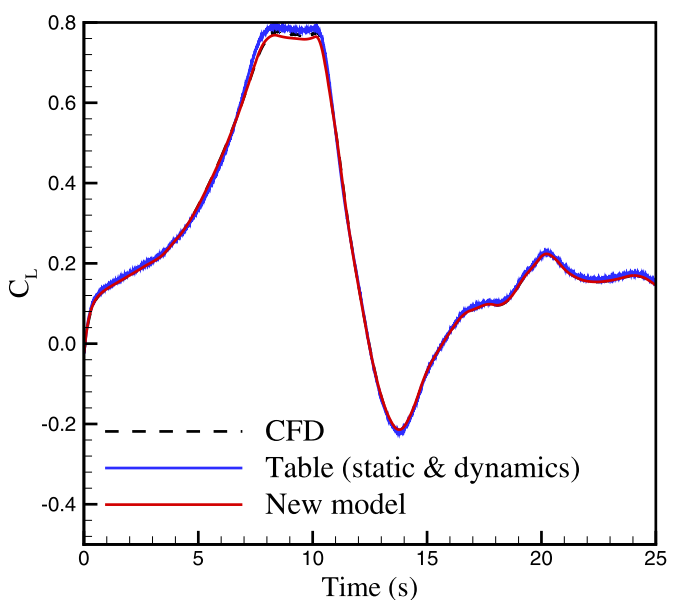

(b) Lift coefficient

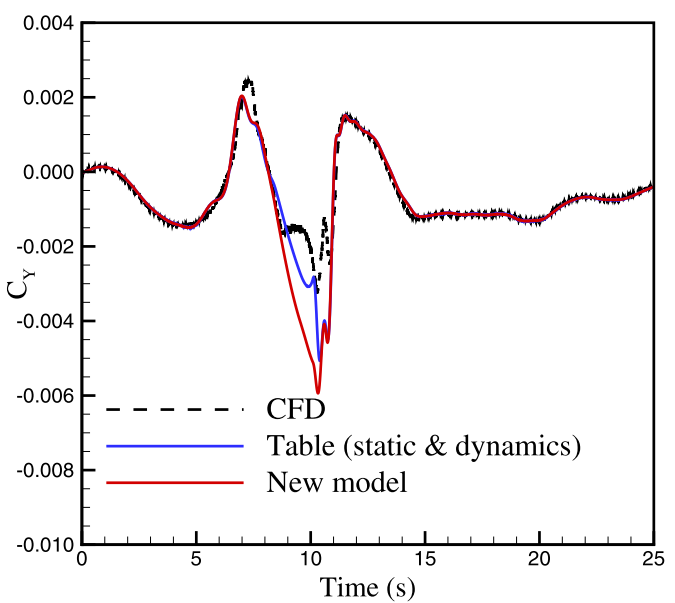

(d) Side-force coefficient

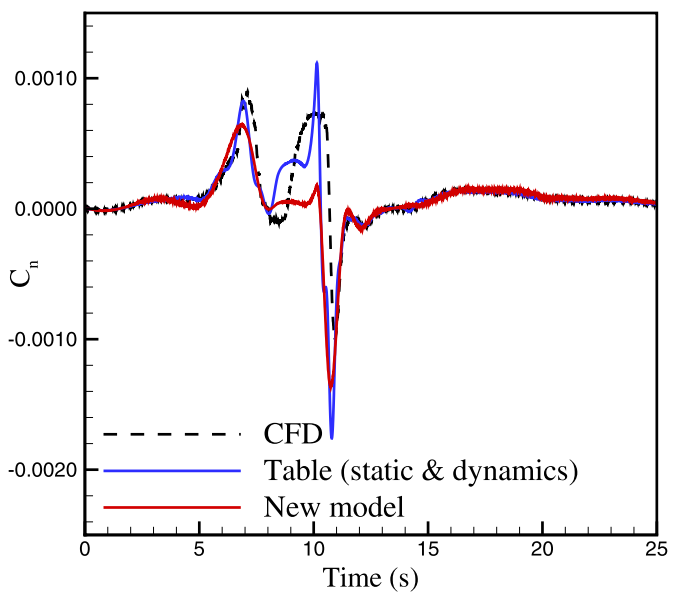

(f) Yaw moment coefficient

Fig. 17. Aerodynamic modeling of Immelmann turn maneuver for angles of attack up to $17^{\circ}$. 
Table 4

Computational cost comparisons.

\begin{tabular}{lc}
\hline & Cost (CPU hrs) \\
\hline Linear ROM & 19000 \\
New ROM & 466000 \\
Barrel roll & 67000 \\
Immelmann turn & 70000 \\
\hline
\end{tabular}

cant financial support for this work that could have influenced its outcome.

\section{Acknowledgements}

Mehdi Ghoreyshi is supported by USAFA under contract FA70001320018; their financial support is gratefully acknowledged. Acknowledgements are expressed to the Department of Defense High Performance Computing Modernization Program for providing computer time. The SACCON geometry was provided by NATO RTO Task Group AVT-161 on Assessment of Stability and Control Prediction Methods for NATO Air \& Sea Vehicles. The authors also appreciate the support provided by the High Performance Computing Research Center at USAFA.

\section{References}

[1] J.T. Betts, Survey of numerical methods for trajectory optimization, J. Guid. Control Dyn. 20 (1) (1997) 57-60.

[2] R.L. Bisplinghoff, H. Ashley, R.L. Halfman, Aeroelasticity, Dover Publications, Mineola, NY, 1996.

[3] K. Brinkman, H.G. Visser, Optimal turn-back maneuvre after engine failure in a single-engine aircraft during climb-out, J. Aerosp. Eng. 22 (G) (2006) 17-27.

[4] C. Canuto, M.Y. Hussaini, A. Quarteroni, T.A. Zang, Spectral Methods in Fluid Dynamics, Springer-Verlag, New York, 1988.

[5] R.M. Cummings, A. Jirásek, M. Ghoreyshi, M.L. Post, Numerical prediction of stability derivatives with various reduced order models, in: AVT-189 Specialists Meeting On Assessment of Stability and Control Prediction Methods for NATO Air and Sea Vehicles, Portsmouth, England, October 2011.

[6] R.M. Cummings, A. Schütte, Integrated computational/experimental approach to unmanned combat air vehicle stability and control estimation, J. Aircr. 49 (6) (2012) 1542-1557.

[7] D.G. Duffy, Advanced Engineering Mathematics with MATLAB, second edition, Chapman and Hall/CRC, Florida, 2003.

[8] B. Etkin, Dynamics of Atmospheric Flight, Dover Publications, New York, 2000.

[9] D. Findeisen, System Dynamics and Mechanical Vibrations: An Introduction, Springer-Verlag, Berlin, 2000.

[10] M. Ghoreyshi, K.J. Badcock, A. Da Ronch, S. Marques, A. Swift, N. Ames, Framework for establishing the limits of tabular aerodynamic models for flight dynamics analysis, J. Aircr. 48 (1) (2011) 42-55, http://dx.doi.org/10.2514/ 1.C001003.

[11] M. Ghoreyshi, K.J. Badcock, M.A. Woodgate, Accelerating the numerical generation of aerodynamic models for flight simulation, J. Aircr. 46 (3) (2009) 972-980.

[12] M. Ghoreyshi, R.M. Cummings, Aerodynamics modeling of a maneuvering aircraft using indicial functions, AIAA paper 2012-689, January 2012.

[13] M. Ghoreyshi, R.M. Cummings, Challenges in the aerodynamics modeling of an oscillating and translating airfoil at large incidence angles, AIAA paper 2010-4392, June-July 2010.

[14] M. Ghoreyshi, A. Jirásek, R.M. Cummings, CFD modeling for trajectory predictions of a generic fighter configuration, AIAA paper 2011-6523, August 2011.

[15] M. Ghoryeshi, A. Jirásek, R.M. Cummings, Computational investigation into the use of response functions for aerodynamic loads modeling, AIAA J. 50 (6) (2012) 1314-1327.

[16] M. Ghoryeshi, M.L. Post, R.M. Cummings, CFD calculation of aerodynamic indicial functions for a generic fighter configuration, ITEA J. Test Eval. 33 (6) (2012) 348-364.

[17] J.J. Gottlieb, C.P.T. Groth, Assessment of Riemann solvers for unsteady onedimensional inviscid flows of perfect gasses, J. Fluids Struct. 78 (2) (1998) 437-458.
[18] D.G. Hull, Conversion of optimal control problems into parameter optimization problems, J. Guid. Control Dyn. 21 (2) (1998) 193-207.

[19] A. Jirásek, R.M. Cummings, SACCON static and dynamic motion flow physics simulations using COBALT, AIAA paper 2011-3965, June 2011.

[20] M.A. Kazemi, M. Razzaghi, The pseudospectral Legendre method for discretizing optimal control problems, IEEE Trans. Autom. Control 40 (10) (1995) $1793-1796$.

[21] V. Klein, P.C. Murphy, Estimation of Aircraft nonlinear unsteady parameters from wind tunnel data, NASA TM-1998-208969, December 1998.

[22] V. Klein, K.D. Norderer, Modeling of aircraft unsteady aerodynamic characteristics, Part I. Postulated models, NASA TM 109120, May 1994.

[23] V. Klein, K.D. Norderer, Modeling of aircraft unsteady aerodynamic characteristics, Part II. Parameters estimated from wind tunnel data, NASA TM 110161, April 1995.

[24] J.G. Leishman, Indicial lift approximations for two-dimensional subsonic flow as obtained from oscillatory measurements, J. Aircr. 30 (3) (1993) 340-351.

[25] J.G. Leishman, T.S. Beddoes, A semi-empirical model for dynamic stall, J. Am. Helicopter Soc. 34 (3) (1989) 3-17.

[26] J.G. Leishman, K.Q. Nguyen, State-space representation of unsteady airfoil behavior, AIAA J. 28 (5) (1989) 836-844.

[27] R. Liebe, Flow Phenomena in Nature: A Challenge to Engineering Design, WIT Press, Southampton, Great Britain, 2007.

[28] P. Lisandrin, G. Carpentieri, M. van Tooren, Investigation over CFD-based models for the identification of nonlinear unsteady aerodynamics responses, AIAA J. 44 (9) (2006) 2043-2050.

[29] T.D. Loeser, D.D. Vicroy, A. Schütte, SACCON static wind tunnel tests at DNWNWB and 14' $\times 22^{\prime}$ NASA LaRC, AIAA Paper 2010-4393, June-July 2010.

[30] D.R. McDaniel, R.M. Cummings, K. Bergeron, S.A. Morton, J.P. Dean, Comparisons of CFD solutions of static and maneuvering fighter aircraft with flight test data, J. Aerosp. Eng. 223 (4) (2009) 323-340.

[31] B.N. Pamadi, P.C. Murphy, V. Klein, J.M. Brandon, Prediction of unsteady aerodynamic coefficients at high angles of attack, AIAA Paper 2001-4077, August 2001.

[32] D.E. Raveh, Reduced-order models for nonlinear unsteady aerodynamics, AIAA J. 39 (8) (2001) 1417-1429.

[33] P.H. Reisenthel, M.T. Bettencourt, Data-based aerodynamic modeling using nonlinear indicial theory, AIAA Paper 99-0763, January 1999.

[34] P.H. Reisenthel, M.T. Bettencourt, Extraction of nonlinear indicial and critical state responses from experimental data, AIAA Paper 99-0764, January 1999.

[35] P.H. Reisenthel, M.T. Bettencourt, J.H. Myatt, D.S. Grismer, A nonlinear indicial prediction tool for unsteady aerodynamic modeling, AIAA Paper 98-4350, August 1998.

[36] D.A. Romero, C. Amon, S. Finger, I. Verdinelli, Multi-stage bayesian surrogates for the design of time-dependent system, in: Proceedings of ASME 2004 Design Engineering Technical Conference and Computers and Information in Engineering, 2004, DETC 2004-57510.

[37] J. Roskam, Airplane Design, Roskam Aviation and Engineering Corporation, Kansas, USA, 1990.

[38] I.M. Ross, F. Fahroo, User's manual for DIDO 2002: a MATLAB application package for dynamic optimization, NPS technical rept, Dept. of Aeronautics and Astronautics, Naval Postgraduate School, 2002, AA-02-002.

[39] A. Schütte, G. Einarsson, A. Raichle, B. Schoning, W. Mönnich, T. Forkert, Numerical simulation of manoeuvreing aircraft by aerodynamic, flight mechanics, and structural mechanics coupling, J. Aircr. 46 (1) (2009) 53-64.

[40] R. Singh, J. Baeder, Direct calculation of three-dimensional indicial lift response using computational fluid dynamics, J. Aircr. 34 (4) (1997) 465-471.

[41] P.R. Spalart, S.R. Allmaras, A one equation turbulence model for aerodynamic flows, AIAA Paper 1992-0439, January 1992.

[42] W.Z. Strang, R.F. Tomaro, M.J. Grismer, The defining methods of cobalt: a parallel, implicit, unstructured Euler/Navier-Stokes flow solver, AIAA paper 1999-0786, 1999.

[43] M. Tobak, On the use of the indicial function concept in the analysis of unsteady motions of wings and wing-tail combinations, NACA report 1188, 1954.

[44] M. Tobak, On nonlinear longitudinal dynamic stability, Technical report in AGARD stability and control, PT. 1, Sep. 1966.

[45] M. Tobak, G.T. Chapman, Nonlinear problems in flight dynamics involving aerodynamic bifurcations, NASA TN-86706, 1985.

[46] M. Tobak, G.T. Chapman, L.B. Schiff, Mathematical modeling of the aerodynamic characteristics in flight dynamics, NASA TM-85880, 1984.

[47] M. Tobak, L.B. Schiff, On the formulation of the aerodynamic characteristics in aircraft dynamics, NASA TR R-456, 1976.

[48] R.F. Tomaro, W.Z. Strang, L.N. Sankar, An implicit algorithm for solving time dependent flows on unstructured grids, AIAA paper 1997-0333, January 1997. 